\title{
A Mathematical Model of Malaria Transmission with Structured Vector Population and Seasonality
}

\author{
Bakary Traoré, Boureima Sangaré, and Sado Traoré \\ Department of Mathematics, Polytechnic University of Bobo Dioulasso, 01 BP 1091, Bobo-Dioulasso 01, Burkina Faso \\ Correspondence should be addressed to Boureima Sangaré; mazou1979@yahoo.fr
}

Received 22 January 2017; Accepted 26 April 2017; Published 4 June 2017

Academic Editor: Sabri Arik

Copyright (C) 2017 Bakary Traoré et al. This is an open access article distributed under the Creative Commons Attribution License, which permits unrestricted use, distribution, and reproduction in any medium, provided the original work is properly cited.

\begin{abstract}
In this paper, we formulate a mathematical model of nonautonomous ordinary differential equations describing the dynamics of malaria transmission with age structure for the vector population. The biting rate of mosquitoes is considered as a positive periodic function which depends on climatic factors. The basic reproduction ratio of the model is obtained and we show that it is the threshold parameter between the extinction and the persistence of the disease. Thus, by applying the theorem of comparison and the theory of uniform persistence, we prove that if the basic reproduction ratio is less than 1 , then the disease-free equilibrium is globally asymptotically stable and if it is greater than 1 , then there exists at least one positive periodic solution. Finally, numerical simulations are carried out to illustrate our analytical results.
\end{abstract}

\section{Introduction}

Malaria is an infectious disease caused by plasmodium parasite which is transmitted to humans through the bites of infectious female mosquitoes. According to the estimations of World Health Organization (WHO) in 2015, 3.2 billion persons were at risk of infection and 2.4 million new cases were detected with 438,000 cases of deaths. However subSaharan Africa remains the most vulnerable region with high rate of deaths due to malaria.

To reduce the impact of malaria in the world, many scientific efforts were done including mathematical models construction. The first model of malaria transmission was developed by Ross [1]. According to Ross, if the mosquito population can be reduced to below a certain threshold, then malaria can be eradicated. Later, Macdonald did some modifications to the model and included superinfection. He showed that reducing the number of mosquitoes has little effect on the epidemiology of malaria in areas of intense transmission [2]. Nowadays, several mathematical models have been developed in order to reduce the malaria death rate in the world $[3,4]$. In spite of the efforts made, it is still difficult to predict future malaria intensity, particularly in view of climate change.
It must be noticed that transmission and distribution of vector-borne diseases are greatly influenced by environmental and climatic factors. Seasonality and circadian rhythm of mosquito population, as well as other ecological and behavioural features, are strongly influenced by climatic factors such as temperature, rainfall, humidity, wind, and duration of daylight [5]. Moreover, in most mathematical models, the mosquito life cycle is generally ignored because eggs, larvae, and pupae are not involved in the transmission cycle. That is a useful simplification of the system but unfortunately the results of these models do not predict malaria intensity in most endemic regions. Thus, it is necessary to consider the life cycle of mosquitoes and the seasonality effect, which are very important aspects of the dynamics of malaria transmission.

Recently, Moulay et al. [6] have formulated a mathematical model describing the mosquito population dynamics which takes into account autoregulation phenomena of eggs and larvae stages. They have defined a threshold and proved that the growth of the mosquito population is governed by that threshold. Considering the climatic factors and the mosquitoes life cycle, we formulate a mathematical model describing the dynamics of malaria transmission. We analyze 
the impact of the model describing the mosquito population dynamics on the model of malaria transmission. Besides, by using the comparison theorem and the theory of uniform persistence, we, respectively, study the global stability of the nontrivial disease-free equilibrium [7-10] and the existence of positive periodic solutions.

This paper is organized as follows. In Section 2, we formulate the mathematical model of our problem. Section 3 provides the mathematical analysis of the model. Computational simulations are performed in Section 4 in order to illustrate our mathematical results. In the last section, Section 5, we conclude and give some remarks and future works.

\section{Model Formulation}

Motivated by the compartmental models in $[6,11]$, we derive an age-structured malaria model with seasonality to account for the cross infection between mosquitoes and humans. The human population is divided into four epidemiological categories representing the state variables: the susceptible class $S_{h}$, exposed class $E_{h}$, infectious class $I_{h}$, and recovered class $R_{h}$ (immune and asymptomatic, but slightly infectious humans). In the life cycle of anopheles, there are mainly two major stages: mature stage and aquatic stage. Therefore, we divide the mosquitoes population into these stages: immature and mature. The immature stage is divided in two compartments: eggs class $E$, larvae and pupae class $L$. In the mature stage, we have three compartments: the susceptible class $S_{m}$, exposed class $E_{m}$, and infectious class $I_{m}$. At any time, the total number of humans and mature mosquitoes is given, respectively, by

$$
\begin{aligned}
N_{h}(t) & =S_{h}(t)+E_{h}(t)+I_{h}(t)+R_{h}(t), \\
A(t) & =S_{m}(t)+E_{m}(t)+I_{m}(t) .
\end{aligned}
$$

It is assumed throughout this paper that

(H1) all vector population measures refer to densities of female mosquitoes,

(H2) the mosquitoes bite only humans,

(H3) there is no vertical transmission of malaria,

(H4) all the new recruits are susceptibles.

2.1. Interactions between Humans and Mosquitoes. When an infectious mosquito bites a susceptible human, the parasite enters the body of the human with a probability $c_{m h}$ and the human moves into the exposed class $E_{h}$. Some time after, he leaves from class $E_{h}$ to class $I_{h}$ with rate $\alpha$. Infectious humans migrate into the class $R_{h}$ after acquisition of their immunity with rate $r_{h}$. The immunized lose their immunity with rate $\gamma$ if they do not have continuous exposure to infection. Humans leave the total population through natural death rate $d_{h}$ and malaria death rate $d_{P}$.

Similarly, when a susceptible mosquito bites an infectious human, it enters the class $E_{m}$ with a probability $c_{h m}$. Some time after, it leaves from class $E_{m}$ to infective class $I_{m}$ with rate $v_{m}$ where it remains for life. Mature mosquitoes leave the population through natural mortality $d_{m}$.
Using the standard incidence as in the model of Ngwa and Shu [4], we define, respectively, the infection incidence from mosquitoes to humans, $k_{h}(t)$, and from humans to mosquitoes, $k_{m}(t)$ :

$$
\begin{aligned}
& k_{h}(t)=c_{m h} \beta(t) \frac{I_{m}(t)}{N_{h}(t)}, \\
& k_{m}(t)=c_{h m} \beta(t) \frac{I_{h}(t)}{N_{h}(t)}+\bar{c}_{h m} \beta(t) \frac{R_{h}(t)}{N_{h}(t)} .
\end{aligned}
$$

Furthermore, using the above assumptions, we obtain the transfer diagram (Figure 1) of the model.

2.2. The Mathematical Model. Using the above assumptions and by making a balance of the movements in each class, we obtain the following system:

$$
\begin{aligned}
& \frac{d E}{d t}(t)=b\left(1-\frac{E(t)}{K_{E}}\right) A(t)-(s+d) E(t), \\
& \frac{d L}{d t}(t)=s\left(1-\frac{L(t)}{K_{L}}\right) E(t)-\left(s_{L}+d_{L}\right) L(t), \\
& \frac{d S_{h}}{d t}(t)=\Lambda+\gamma R_{h}(t)-\left(d_{h}+k_{h}(t)\right) S_{h}(t), \\
& \frac{d E_{h}}{d t}(t)=k_{h}(t) S_{h}(t)-\left(d_{h}+\alpha\right) E_{h}(t), \\
& \frac{d I_{h}}{d t}(t)=\alpha E_{h}(t)-\left(d_{h}+d_{p}+r_{h}\right) I_{h}(t), \\
& \frac{d R_{h}}{d t}(t)=r_{h} I_{h}(t)-\left(d_{h}+\gamma\right) R_{h}(t), \\
& \frac{d S_{m}}{d t}(t)=s_{L} L(t)-\left(d_{m}+k_{m}(t)\right) S_{m}(t), \\
& \frac{d E_{m}}{d t}(t)=k_{m}(t) S_{m}(t)-\left(v_{m}+d_{m}\right) E_{m}(t), \\
& \frac{d I_{m}}{d t}(t)=v_{m} E_{m}(t)-d_{m} I_{m}(t) .
\end{aligned}
$$

The growth of the whole human population and mature vector is, respectively, described by the following equations:

$$
\begin{aligned}
& \frac{d N_{h}}{d t}(t)=\Lambda-d_{h} N_{h}(t)-d_{p} I_{h}(t), \\
& \frac{d A}{d t}(t)=s_{L} L(t)-d_{m} A(t) .
\end{aligned}
$$




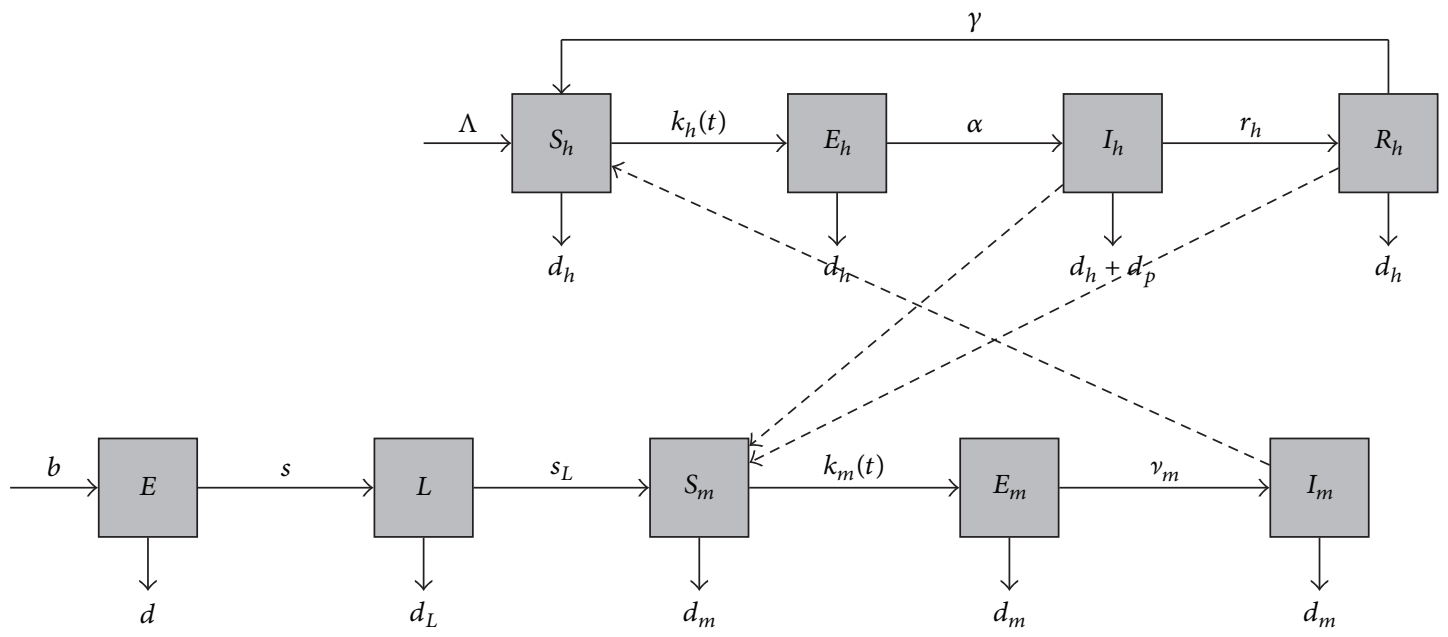

FIGURE 1: The dashed arrows indicate the direction of the infection and the solid arrows represent the transition from one class to another.

Using (2), we get $S_{m}(t)=A(t)-E_{m}(t)-I_{m}(t)$ and then the model can be rewritten as follows:

$$
\begin{aligned}
& \frac{d E}{d t}(t)=b\left(1-\frac{E(t)}{K_{E}}\right) A(t)-(s+d) E(t), \\
& \frac{d L}{d t}(t)=s\left(1-\frac{L(t)}{K_{L}}\right) E(t)-\left(s_{L}+d_{L}\right) L(t), \\
& \frac{d A}{d t}(t)=s_{L} L(t)-d_{m} A(t), \\
& \frac{d S_{h}}{d t}(t)=\Lambda+\gamma R_{h}(t)-\left(d_{h}+k_{h}(t)\right) S_{h}(t), \\
& \frac{d E_{h}}{d t}(t)=k_{h}(t) S_{h}(t)-\left(d_{h}+\alpha\right) E_{h}(t), \\
& \frac{d I_{h}}{d t}(t)=\alpha E_{h}(t)-\left(d_{h}+d_{p}+r_{h}\right) I_{h}(t), \\
& \frac{d R_{h}}{d t}(t)=r_{h} I_{h}(t)-\left(d_{h}+\gamma\right) R_{h}(t), \\
& \frac{d E_{m}}{d t}(t)=k_{m}(t) \mathrm{A}(t)-k_{m}(t) I_{m}(t) \\
& \quad(t)=v_{m} E_{m}(t)-d_{m} I_{m}(t) . \\
& \left.\frac{d t}{d t}+k_{m}(t)\right) E_{m}(t),
\end{aligned}
$$

Mathematically model (7) can be written as follows:

$$
\dot{X}(t)=F(t, X(t)),
$$

where $X(t)=\left(E(t), L(t), A(t), S_{h}(t), E_{h}(t), I_{h}(t), R_{h}(t), E_{m}(t)\right.$, $\left.I_{m}(t)\right)^{T}$. The function $F: \mathbb{R}_{+} \times \mathbb{R}^{9} \rightarrow \mathbb{R}^{9}$ is $C^{\infty}\left(\mathbb{R}^{9}\right)$ and defined by

$$
\begin{aligned}
& F(t, X(t)) \\
& =\left(\begin{array}{c}
b\left(1-\frac{E(t)}{K_{E}}\right) A(t)-(s+d) E(t) \\
s\left(1-\frac{L(t)}{K_{L}}\right) E(t)-\left(s_{L}+d_{L}\right) L(t) \\
s_{L} L(t)-d_{m} A(t) \\
\Lambda+\gamma R_{h}(t)-\left(d_{h}+k_{h}(t)\right) S_{h}(t) \\
k_{h}(t) S_{h}(t)-\left(d_{h}+\alpha\right) E_{h}(t) \\
\alpha E_{h}(t)-\left(d_{h}+d_{p}+r_{h}\right) I_{h}(t) \\
r_{h} I_{h}(t)-\left(d_{h}+\gamma\right) R_{h}(t) \\
k_{m}(t) A(t)-k_{m}(t) I_{m}(t)-\left(v_{m}+d_{m}+k_{m}(t)\right) E_{m}(t) \\
v_{m} E_{m}(t)-d_{m} I_{m}(t)
\end{array}\right)
\end{aligned}
$$

Let us consider $F=\left(F_{1}, F_{2}\right)^{T}$ and $X(t)=\left(X_{1}(t), X_{2}(t)\right)^{T}$ with $X_{1}(t)=(E(t), L(t), A(t))^{T}$ and $X_{2}(t)=\left(S_{h}(t), E_{h}(t), I_{h}(t)\right.$, $\left.R_{h}(t), E_{m}(t), I_{m}(t)\right)^{T}$. Then system (8) can be rewritten as follows:

$$
\begin{aligned}
& \dot{X}_{1}(t)=F_{1}\left(X_{1}(t), X_{2}(t)\right), \\
& \dot{X}_{2}(t)=F_{2}\left(t, X_{1}(t), X_{2}(t)\right),
\end{aligned}
$$

with the functions $F_{1}$ and $F_{2}$ defined as follows:

$$
\begin{aligned}
& F_{2}\left(t, X_{1}(t), X_{2}(t)\right) \\
& =\left(\begin{array}{c}
\Lambda+\gamma R_{h}(t)-\left(d_{h}+k_{h}(t)\right) S_{h}(t) \\
k_{h}(t) S_{h}(t)-\left(d_{h}+\alpha\right) E_{h}(t) \\
\alpha E_{h}(t)-\left(d_{h}+d_{p}+r_{h}\right) I_{h}(t) \\
r_{h} I_{h}(t)-\left(d_{h}+\gamma\right) R_{h}(t) \\
k_{m}(t) A(t)-k_{m}(t) I_{m}(t)-\left(v_{m}+d_{m}+k_{m}(t)\right) E_{m}(t) \\
v_{m} E_{m}(t)-d_{m} I_{m}(t)
\end{array}\right),
\end{aligned}
$$




$$
F_{1}\left(X_{1}(t), X_{2}(t)\right)=\left(\begin{array}{c}
b\left(1-\frac{E(t)}{K_{E}}\right) A(t)-(s+d) E(t) \\
s\left(1-\frac{L(t)}{K_{L}}\right) E(t)-\left(s_{L}+d_{L}\right) L(t) \\
s_{L} L(t)-d_{m} A(t)
\end{array}\right) .
$$

System (10) describes the maturation cycle of mosquitoes and system (11) describes the dynamics of malaria transmission. System (10) is biologically well defined in

$$
\Delta:=\left\{(E, L, A) \in \mathbb{R}_{+}^{3} E \leq K_{E}, L \leq K_{L}, A \leq \frac{s_{L}}{d_{m}} K_{L}\right\}
$$

and system (11) is biologically well defined in

$$
\begin{aligned}
\Omega= & \left\{\left(S_{h}, E_{h}, I_{h}, R_{h}, E_{m}, I_{m}\right) \in \mathbb{R}_{+}^{6} \mid\right. \\
& \left.S_{h}+E_{h}+I_{h}+R_{h} \leq \frac{\Lambda}{d_{h}}, E_{m}+I_{m} \leq \frac{s_{L} K_{L}}{d_{m}}\right\} ;
\end{aligned}
$$

then model (7) is biologically well defined in $\Gamma:=\Delta \times \Omega$.

\section{Mathematical Analysis}

\subsection{Positivity and Boundedness of Solutions}

Lemma 1 (see [6]). The set $\Delta$ is a positive invariant region under the flow induced by (10).

We assume that

(H5) $\beta(t)$ is a $\omega$-periodic positive function with $\omega=12$ months,

(H6) all the parameters of the model are positive except the disease-induced death rate, $d_{p}$, which is assumed to be nonnegative.

Theorem 2. For any initial condition $\phi \in \mathbb{R}_{+}^{9}$, system (8) has a unique solution. Further, the compact $\Gamma$ is a positively invariant set, which attracts all positive orbits in $\mathbb{R}_{+}^{9}$.

Proof. For all $\phi \in \mathbb{R}_{+}^{9}$, the function $F$ is locally Lipschitzian in $X(t)$. It then follows through Cauchy-Lipschitz theorem that system (8) has a unique local solution.

Furthermore, according to (6), we have

$$
\begin{gathered}
\frac{d N_{h}}{d t}(t)=\Lambda-d_{h} N_{h}(t)-d_{p} I_{h}(t) \leq \Lambda-d_{h} N_{h}(t), \\
\frac{d A}{d t}(t)=s_{L} L(t)-d_{m} A(t) \leq s_{L} K_{L}-d_{m} A(t) .
\end{gathered}
$$

It then follows that if $N_{h}(t)>\Lambda / d_{h}$ and $A(t)>s_{L} K_{L} / d_{m}$, then $d N_{h} / d t(t)<0$ and $d A / d t(t)<0$.

Let us consider the following differential equations:

$$
\begin{aligned}
\frac{d N_{h}}{d t}(t) & =\Lambda-d_{h} N_{h}(t), \\
\frac{d A}{d t}(t) & =s_{L} K_{L}-d_{m} A(t)
\end{aligned}
$$

with general solutions:

$$
\begin{aligned}
N_{h}(t) & =\frac{\Lambda}{d_{h}}+\left(N_{h}(0)-\frac{\Lambda}{d_{h}}\right) e^{-d_{h} t}, \\
A(t) & =\frac{s_{L} K_{L}}{d_{m}}+\left(A(0)-\frac{s_{L} K_{L}}{d_{m}}\right) e^{-d_{m} t} .
\end{aligned}
$$

By applying the standard comparison theorem, we obtain, for all $t \geq 0, N_{h}(t) \leq \Lambda / d_{h}$ and $A(t) \leq s_{L} K_{L} / d_{m}$ if $N_{h}(0)$ $\leq \Lambda / d_{h}$ and $A(0) \leq s_{L} K_{L} / d_{m}$. Thus, the set $\Omega$ is positively invariant with respect to system (11). Therefore, from Lemma 1 , the set $\Delta$ is positively invariant with respect to system (10). Then, we conclude that the compact set $\Gamma=\Delta \times \Omega$ is positively invariant. Thus, all the solutions of system (8) are nonnegative and bounded.

3.2. Disease-Free Equilibriums. Let us consider the following threshold parameter: $r=(b /(s+d))\left(s /\left(s_{L}+d_{L}\right)\right)\left(s_{L} / d_{m}\right)$. Then we have the following result.

Proposition 3 (see [6]). System (10) always has the mosquitofree equilibrium $P_{0}=(0,0,0)$.

(i) If $r \leq 1$, then system (10) has no other equilibrium.

(ii) If $r>1$, there is a unique endemic equilibrium

$$
P_{1}=\left(E^{*}, L^{*}, A^{*}\right)=\left(1-\frac{1}{r}\right)\left(\frac{K_{E}}{\gamma_{E}}, \frac{K_{L}}{\gamma_{L}}, \frac{s_{L}}{d_{m}} \frac{K_{L}}{\gamma_{L}}\right) \text {, }
$$

where

$$
\begin{aligned}
& \gamma_{E}=1+\frac{(s+d) d_{m} K_{E}}{b s_{L} K_{L}}, \\
& \gamma_{L}=1+\frac{\left(s_{L}+d_{L}\right) K_{L}}{s K_{E}} .
\end{aligned}
$$

Lemma 4. Model (7) has

(i) trivial disease-free equilibrium $E_{0}=\left(0,0,0, S_{h}^{*}, 0,0,0\right.$, $0,0)$ if $r \leq 1$,

(ii) nontrivial disease-free equilibrium $E_{1}=\left(E^{*}, L^{*}, A^{*}\right.$, $\left.S_{h}^{*}, 0,0,0,0,0\right)$ if $r>1$, where $S_{h}^{*}=\Lambda / d_{h}, A^{*}=S_{m}^{*}=$ $s_{L} L^{*} / d_{m}$, and $E^{*}, L^{*}$, and $A^{*}$ are given above.

Proof. By solving the system $F_{2}\left(t, X_{1}(t), X_{2}(t)\right)=0$ at the disease-free equilibrium, $E_{h}(t)=I_{h}(t)=R_{h}(t)=E_{m}(t)=$ $I_{m}(t)=0, \forall t \geq 0$, we get the equilibrium point $E_{1}^{+}=$ $\left(S_{h}^{*}, 0,0,0,0,0\right)$ for system (11), with $S_{h}^{*}=\Lambda / d_{h}$. Moreover, thanks to Proposition 3, system (10) has a unique mosquitofree equilibrium $(0,0,0)$ if $r \leq 1$ and a unique endemic equilibrium $\left(E^{*}, L^{*}, A^{*}\right)$ if $r>1$. Thus, we conclude that system (7) has a trivial disease-free equilibrium $E_{0}=(0,0,0$, $\left.S_{h}^{*}, 0,0,0,0,0\right)$ if $r \leq 1$ and a nontrivial disease-free equilibrium $E_{1}=\left(E^{*}, L^{*}, A^{*}, S_{h}^{*}, 0,0,0,0,0\right)$ if $r>1$.

Remark 5. We will only consider the equilibrium state $E_{1}$ because it is more biologically realistic. So, in the rest of the paper, we assume that $r>1$. 
3.3. Threshold Dynamics. Linearizing system (8) at the equilibrium state $E_{1}$, we obtain the following system (here we write down only the equations for the "diseased" classes):

$$
\begin{aligned}
\frac{d E_{h}}{d t}(t)= & c_{m h} \beta(t) I_{m}(t)-\left(d_{h}+\alpha\right) E_{h}(t), \\
\frac{d I_{h}}{d t}(t)= & \alpha E_{h}(t)-\left(d_{h}+d_{p}+r_{h}\right) I_{h}(t), \\
\frac{d R_{h}}{d t}(t)= & r_{h} I_{h}(t)-\left(d_{h}+\gamma\right) R_{h}(t), \\
\frac{d E_{m}}{d t}(t)= & c_{h m} \beta(t) \frac{A^{*}}{S_{h}^{*}} I_{h}(t)+\bar{c}_{h m} \beta(t) \frac{A^{*}}{S_{h}^{*}} R_{h}(t) \\
& -\left(\nu_{m}+d_{m}\right) E_{m}(t), \\
\frac{d I_{m}}{d t}(t)= & v_{m} E_{m}(t)-d_{m} I_{m}(t) .
\end{aligned}
$$

This system can be rewritten as

$$
\frac{d Z(t)}{d t}=(F(t)-V(t)) Z(t)
$$

where $Z(t)=\left(E_{h}(t), I_{h}(t), R_{h}(t), E_{m}(t), I_{m}(t)\right)^{T}$ and $F(t)$ and $V(t)$ are $5 \times 5$ matrix defined as follows:

$$
\begin{aligned}
& F(t) \\
& =\left(\begin{array}{ccccc}
0 & 0 & 0 & 0 & c_{m h} \beta(t) \\
0 & 0 & 0 & 0 & 0 \\
0 & 0 & 0 & 0 & 0 \\
0 & c_{h m} \beta(t) \frac{A^{*}}{S_{h}^{*}} \bar{c}_{h \mathrm{~m}} \beta(t) \frac{A^{*}}{S_{h}^{*}} & 0 & 0 \\
0 & 0 & 0 & 0 & 0
\end{array}\right),
\end{aligned}
$$

$V(t)$

$$
=\left(\begin{array}{ccccc}
d_{h}+\alpha & 0 & 0 & 0 & 0 \\
-\alpha & d_{h}+d_{p}+r_{h} & 0 & 0 & 0 \\
0 & -r_{h} & d_{h}+\gamma & 0 & 0 \\
0 & 0 & 0 & v_{m}+d_{m} & 0 \\
0 & 0 & 0 & -v_{m} & d_{m}
\end{array}\right) .
$$

Let us assume that $Y(t, s), t \geq s$, is the matrix solution of the linear $\omega$-periodic system

$$
\frac{d y}{d t}=-V(t) y
$$

That is, for each $s \in \mathbb{R}$, the $5 \times 5$ matrix $Y(t, s)$ satisfies the equation

$$
\frac{d}{d t} Y(t, s)=-V(t) Y(t, s), \quad \forall t \geq s, Y(s, s)=I,
$$

where $I$ is the $5 \times 5$ identity matrix. Thus, the monodromy matrix $\Phi_{-V}(t)$ of $(23)$ is equal to $Y(t, 0), \forall t \geq 0$.
Let $C_{\omega}$ be the ordered Banach space of all $\omega$-periodic functions from $\mathbb{R}$ to $\mathbb{R}^{5}$ which is equipped with the maximum norm $\|\cdot\|$ and the positive cone $C_{\omega}^{+}:=\left\{\phi \in \mathrm{C}_{\omega}: \phi(t) \geq 0, \forall t \in\right.$ $\mathbb{R}\}$. Then, we can define a linear operator $\mathscr{L}: C_{\omega} \rightarrow C_{\omega}$ by

$$
(\mathscr{L} \phi)(t)=\int_{0}^{\infty} Y(t, t-a) F(t-a) \phi(t-a) d a,
$$

$$
\forall t \in \mathbb{R}, \phi \in C_{\omega} .
$$

It then follows from [12] that $\mathscr{L}$ is the next infection operator, and the basic reproduction ratio is $\mathscr{R}_{0}=\rho(\mathscr{L})$, the spectral radius of $\mathscr{L}$.

In order to calculate $\mathscr{R}_{0}$, we consider the following linear $\omega$-periodic system:

$$
\frac{d w(t)}{d t}=\left[\frac{1}{\lambda} F(t)-V(t)\right] w(t)
$$

$$
\forall t \in \mathbb{R}_{+}, \lambda \in(0, \infty) \text {. }
$$

Let $W(t, s, \lambda), t \geq s, s \in \mathbb{R}$, be the evolution operator of system (26) on $\mathbb{R}^{5}$. Clearly $W(t, 0,1)=\Phi_{F-V}(t), \forall t \geq 0$. The following result will be used in our numerical calculation of the basic reproduction ratio.

Lemma 6 (see [12]). (i) If $\rho(W(\omega, 0, \lambda))=1$ has a positive solution $\lambda_{0}$, then $\lambda_{0}$ is an eigenvalue of $\mathscr{L}$, and hence $\mathscr{R}_{0}>0$.

(ii) If $\mathscr{R}_{0}>0$, then $\lambda=\mathscr{R}_{0}$ is the unique solution of $\rho(W(\omega, 0, \lambda))=1$.

(iii) $\mathscr{R}_{0}=0$ if and only if $\rho(W(\omega, 0, \lambda))<1$, for all $\lambda>0$.

3.4. Stability of Equilibrium State $E_{1}$. In this section, we will study the asymptotic behaviour of the nontrivial equilibrium $E_{1}$; thus we have the following result, which will be used in the proofs of our main results.

Lemma 7 (see [12]). The following statements are valid:

(i) $\mathscr{R}_{0}=1$ if and only if $\rho\left(\Phi_{F-V}(\omega)\right)=1$.

(ii) $\mathscr{R}_{0}<1$ if and only if $\rho\left(\Phi_{F-V}(\omega)\right)<1$.

(iii) $\mathscr{R}_{0}>1$ if and only if $\rho\left(\Phi_{F-V}(\omega)\right)>1$.

Lemma 8 (see [6]). If $r>1$, then $P_{1}$ is globally asymptotically stable in $\operatorname{int}(\Delta)$, with respect to system (10).

Theorem 9. The nontrivial equilibrium $E_{1}$ is locally asymptotically stable if $\mathscr{R}_{0}<1$ and unstable if $\mathscr{R}_{0}>1$.

Proof. Let $\mathscr{A}(t)$ be the Jacobian matrix of (8) evaluated at $E_{1}$. Then we have

$$
\mathscr{A}(t)=\left(\begin{array}{ll}
\mathscr{A}_{11} & \mathscr{A}_{12} \\
\mathscr{A}_{21} & \mathscr{A}_{22}(t)
\end{array}\right),
$$


where

$$
\begin{aligned}
& \mathscr{A}_{12}=\left(\begin{array}{cccccc}
0 & 0 & 0 & 0 & b\left(1-\frac{E^{*}}{K_{E}}\right) & b\left(1-\frac{E^{*}}{K_{E}}\right) \\
0 & 0 & 0 & 0 & 0 & 0 \\
0 & 0 & 0 & 0 & -d_{m} & -d_{m}
\end{array}\right), \\
& \mathscr{A}_{21}=\left(\begin{array}{lll}
0 & 0 & 0 \\
0 & 0 & 0 \\
0 & 0 & 0 \\
0 & 0 & 0 \\
0 & 0 & 0 \\
0 & 0 & 0
\end{array}\right), \\
& \mathscr{A}_{11} \\
& =\left(\begin{array}{ccc}
-(s+d)-\frac{b A^{*}}{K_{E}} & 0 & (s+d) \frac{E^{*}}{A^{*}} \\
\left(s_{L}+d_{L}\right) \frac{L^{*}}{E^{*}} & -\left(s_{L}+d_{L}\right)-\frac{s E^{*}}{K_{L}} & 0 \\
0 & s_{L} & -d_{m}
\end{array}\right), \\
& \mathscr{A}_{22}(t)=\left(\begin{array}{cc}
-d_{h} & C(t) \\
\widehat{0} & F(t)-V(t)
\end{array}\right)
\end{aligned}
$$

with

$$
\begin{aligned}
C(t) & =\left(\begin{array}{lllll}
0 & 0 & \gamma & 0 & -c_{m h} \beta(t)
\end{array}\right), \\
\widehat{0} & =(0,0,0,0,0)^{T} .
\end{aligned}
$$

$E_{1}$ is locally asymptotically stable if $\rho\left(\Phi_{\mathscr{A}}(\omega)\right)<1$. The matrix $\mathscr{A}_{11}$ is a constant matrix and its characteristic equation is given by $\pi(z)=z^{3}+a_{1} z^{2}+a_{2} z+a_{3}$, where

$$
\begin{aligned}
a_{1} & =\left(1-\frac{1}{r}\right)\left(\frac{s K_{E}}{\gamma_{E} K_{L}}+\frac{b s_{L} K_{L}}{d_{m} \gamma_{L} K_{E}}\right)+s_{L}+d_{L}+s+d \\
& +d_{m} \\
a_{2} & =\left[\frac{b s_{L} K_{L}}{d_{m} \gamma_{L} K_{E}}\left(1-\frac{1}{r}\right)\right]\left[\frac{s K_{E}}{\gamma_{E} K_{L}}\left(1-\frac{1}{r}\right)+s_{L}+d_{L}\right. \\
& \left.+d_{m}\right]+\frac{d_{m} s K_{E}}{\gamma_{E} K_{L}}\left(1-\frac{1}{r}\right)+d_{m}\left(s_{L}+d_{L}\right), \\
a_{3} & =d_{m}\left(1-\frac{1}{r}\right)\left[\frac{b s s_{L}}{d_{m} \gamma_{L} \gamma_{E}}\left(1-\frac{1}{r}\right)+(s+d) \frac{s K_{E}}{\gamma_{E} K_{L}}\right. \\
& \left.+\left(s_{L}+d_{L}\right) \frac{b s_{L} K_{L}}{d_{m} \gamma_{L} K_{E}}\right] .
\end{aligned}
$$

If $r>1$, then $a_{1}, a_{2}, a_{3}$ and $a_{1} a_{2}-a_{3}$ are clearly positive. So, thanks to Routh-Hurwitz criterion, all eigenvalues of $\mathscr{A}_{11}$ have negative real part. It then follows that $\rho\left(\Phi_{\mathscr{A}_{11}}(\omega)\right)<1$. Thus, the stability of $E_{1}$ depends on $\Phi_{\mathscr{A}_{22}}(\omega)$.

Thus, if $\rho\left(\Phi_{F-V}(\omega)\right)<1$, then $\rho\left(\Phi_{\mathscr{A}_{22}}(\omega)\right)<1$ and then $E_{1}$ is stable. If $\rho\left(\Phi_{F-V}(\omega)\right)>1$ then $E_{1}$ is unstable. So, thanks to Lemma 7, $E_{1}$ is locally asymptotically stable if $\mathscr{R}_{0}<1$ and unstable if $\mathscr{R}_{0}>1$.
Lemma 10 (see [13]). Let $\theta=(1 / \omega) \ln \rho\left(\Phi_{A(\cdot)}(\omega)\right)$; then there exists a positive $\omega$-periodic function $v(t)$ such that $e^{\theta t} v(t)$ is a solution of $\dot{x}(t)=A(t) x(t)$.

Theorem 11. If $\mathscr{R}_{0}<1$ and $d_{p}=0$, then $E_{1}$ is globally asymptotically stable.

Proof. If $d_{p}=0$, we can rewrite (6) as follows:

$$
\begin{aligned}
\frac{d N_{h}}{d t}(t) & =\Lambda-d_{h} N_{h}(t), \\
\frac{d A}{d t}(t) & =s_{L} L(t)-d_{m} A(t) .
\end{aligned}
$$

Thus, there exists a period $\omega^{\prime}$ such that $\forall t \geq \omega^{\prime}, N_{h}(t) \geq N_{h}^{*}-$ $\epsilon$ and $A(t) \leq A^{*}+\epsilon, \forall \epsilon>0$.

At disease-free equilibrium, we have $N_{h}^{*}=S_{h}^{*}$ and $S_{m}^{*}=$ $A^{*}$. So, $A(t) / N_{h}(t) \leq\left(A^{*}+\epsilon\right) /\left(S_{h}^{*}-\epsilon\right)$. It then follows from system (11) that

$$
\begin{aligned}
\frac{d E_{h}}{d t}(t) \leq & c_{m h} \beta(t) I_{m}(t)-\left(d_{h}+\alpha\right) E_{h}(t), \\
\frac{d I_{h}}{d t}(t)= & \alpha E_{h}(t)-\left(d_{h}+r_{h}\right) I_{h}(t), \\
\frac{d R_{h}}{d t}(t)= & r_{h} I_{h}(t)-\left(d_{h}+\gamma\right) R_{h}(t), \\
\frac{d E_{m}}{d t}(t) \leq & c_{h m} \beta(t) \frac{A^{*}+\epsilon}{S_{h}^{*}-\epsilon} I_{h}(t) \\
& +\bar{c}_{h m} \beta(t) \frac{A^{*}+\epsilon}{S_{h}^{*}-\epsilon} R_{h}(t) \\
& \quad\left(v_{m}+d_{m}\right) E_{m}(t) \\
\frac{d I_{m}}{d t}(t)= & v_{m} E_{m}(t)-d_{m} I_{m}(t) .
\end{aligned}
$$

Let us consider the following auxiliary system:

$$
\begin{aligned}
\frac{d \bar{E}_{h}}{d t}(t)= & c_{m h} \beta(t) \bar{I}_{m}(t)-\left(d_{h}+\alpha\right) \bar{E}_{h}(t), \\
\frac{d \bar{I}_{h}}{d t}(t)= & \alpha \bar{E}_{h}(t)-\left(d_{h}+r_{h}\right) \bar{I}_{h}(t), \\
\frac{d \bar{R}_{h}}{d t}(t)= & r_{h} \bar{I}_{h}(t)-\left(d_{h}+\gamma\right) \bar{R}_{h}(t), \\
\frac{d \bar{E}_{m}}{d t}(t)= & c_{h m} \beta(t) \frac{A^{*}+\epsilon}{S_{h}^{*}-\epsilon} \bar{I}_{h}(\mathrm{t}) \\
& +\bar{c}_{h m} \beta(t) \frac{A^{*}+\epsilon}{S_{h}^{*}-\epsilon} \bar{R}_{h}(t) \\
& -\left(v_{m}+d_{m}\right) \bar{E}_{m}(t), \\
\frac{d \bar{I}_{m}}{d t}(t)= & v_{m} \bar{E}_{m}(t)-d_{m} \bar{I}_{m}(t),
\end{aligned}
$$

which can be rewritten as follows:

$$
\begin{aligned}
\frac{d \bar{h}}{d t}(t)= & M_{\epsilon}(t) \bar{h}(t) ; \\
& \bar{h}(t)=\left(\bar{E}_{h}(t), \bar{I}_{h}(t), \bar{R}_{h}(t), \bar{E}_{m}(t), \bar{I}_{m}(t)\right)^{T}
\end{aligned}
$$


with

$$
M_{\epsilon}(t)=\left(\begin{array}{ccccc}
-\left(d_{h}+\alpha\right) & 0 & 0 & 0 & c_{m h} \beta(t) \\
\alpha & -\left(d_{h}+r_{h}\right) & 0 & 0 & 0 \\
0 & r_{h} & -\left(d_{h}+\gamma\right) & 0 & 0 \\
0 & c_{h m} \beta(t) \frac{A^{*}+\epsilon}{S_{h}^{*}-\epsilon} & \bar{c}_{h m} \beta(t) \frac{A^{*}+\epsilon}{S_{h}^{*}-\epsilon} & -\left(v_{m}+d_{m}\right) & 0 \\
0 & 0 & 0 & v_{m} & -d_{m}
\end{array}\right) .
$$

From Lemma 7, if $\mathscr{R}_{0}<1$, then $\rho\left(\Phi_{F-V}(\omega)\right)<1$. Clearly, $\lim _{\epsilon \rightarrow 0^{+}} \Phi_{M_{e}}(\omega)=\Phi_{F-V}(\omega)$ and, by continuity of the spectral radius, we have $\lim _{\epsilon \rightarrow 0^{+}} \rho\left(\Phi_{M_{\epsilon}}(\omega)\right)=\rho\left(\Phi_{F-V}(\omega)\right)<1$. Thus, there exists $\epsilon_{1}>0$ such that $\rho\left(\Phi_{M_{\epsilon}}(\omega)\right)<1, \forall \epsilon \in\left[0, \epsilon_{1}[\right.$.

From Lemma 10, there exists a positive $\omega$-periodic function $v(t)$ such that $\bar{h}(t)=e^{\theta t} v(t)$ is a solution of (34). Since $\rho\left(\Phi_{M_{\varepsilon}}(\omega)\right)<1, \theta<0$. The $\omega$-periodic function $v(t)$ is bounded and it then follows that $\lim _{t \rightarrow \infty} \bar{h}(t)=0$. Applying comparison theorem on system (32a)-(32e), we get $\lim _{t \rightarrow \infty}\left(E_{h}(t), I_{h}(t), R_{h}(t), E_{m}(t), I_{m}(t)\right)=(0,0,0,0,0)$. Using the theory of asymptotically periodic semiflow [[14], Theorem 3.2.1], we have $\lim _{t \rightarrow \infty} S_{h}(t)=S_{h}^{*}, \lim _{t \rightarrow \infty} A(t)=$ $A^{*}=S_{m}^{*}$. From Lemma 8 , if $r>1$ then $P_{1}$ is globally asymptotically stable, so $\lim _{t \rightarrow \infty} E(t)=E^{*}$ and $\lim _{t \rightarrow \infty} L(t)=$ $L^{*}$. Hence, the equilibrium $E_{1}$ is globally attractive.

3.5. Existence of Positive Periodic Solutions. System (8) is constructed by coupling two subsystems. The term coupling these two systems is given by the function $s_{L} L(t)$. The coupling takes place only in one direction because the dynamics of system (11) depend on the dynamics of system (10). The asymptotic behaviour of system (10) is given by Lemma 8 . Now we are going to study the existence of positive periodic solutions of system (11):

$$
\begin{aligned}
\frac{d S_{h}}{d t}(t)= & \Lambda+\gamma R_{h}(t)-\left(d_{h}+k_{h}(t)\right) S_{h}(t), \\
\frac{d E_{h}}{d t}(t)= & k_{h}(t) S_{h}(t)-\left(d_{h}+\alpha\right) E_{h}(t), \\
\frac{d I_{h}}{d t}(t)= & \alpha E_{h}(t)-\left(d_{h}+d_{p}+r_{h}\right) I_{h}(t), \\
\frac{d R_{h}}{d t}(t)= & r_{h} I_{h}(t)-\left(d_{h}+\gamma\right) R_{h}(t), \\
\frac{d E_{m}}{d t}(t)= & k_{m}(t) A(t)-k_{m}(t) I_{m}(t) \\
& \quad-\left(v_{m}+d_{m}+k_{m}(t)\right) E_{m}(t), \\
\frac{d I_{m}}{d t}(t)= & v_{m} E_{m}(t)-d_{m} I_{m}(t) .
\end{aligned}
$$

Model (11) is well defined in $\Omega$ and if $r>1$ it has a disease-free equilibrium $E_{1}^{+}=\left(S_{h}^{*}, 0,0,0,0,0\right)$ with $S_{h}^{*}=\Lambda / d_{h}$.
Let us consider the following sets:

$$
\begin{aligned}
X & :=\mathbb{R}_{+}^{6}, \\
X_{0} & :=\left\{\left(S_{h}, E_{h}, I_{h}, R_{h}, E_{m}, I_{m}\right) \in X \mid E_{h}>0, I_{h}\right. \\
& \left.>0, R_{h}>0, E_{m}>0, I_{m}>0\right\}, \\
\partial X_{0} & :=X \backslash X_{0} .
\end{aligned}
$$

Let $u(t, \psi)$ be the unique solution of (11) with initial conditions $\psi, \Phi(t)$ the periodic semiflow generated by periodic system (11), and $P: X \rightarrow X$ the Poincare map associated with system (11); namely,

$$
\begin{gathered}
P(\psi)=\Phi(\omega) \psi=u(\omega, \psi), \quad \forall \psi \in X, \\
P^{m}(\psi)=\Phi(m \omega) \psi=u(m \omega, \psi), \quad \forall m \geq 0 .
\end{gathered}
$$

Proposition 12. The sets $X_{0}$ and $\partial X_{0}$ are positively invariant under the flow induced by (11).

Proof. Note that if $X_{0}$ is positively invariant, then $\partial X_{0}$ is positively invariant. Thus we only need to prove that $X_{0}$ is positively invariant.

For any initial condition $\psi \in X_{0}$, solving the equations of system (11) we derive that

$$
\begin{aligned}
& S_{h}(t)=\exp \left(-\int_{0}^{t}\left(k_{h}(s)+d_{h}\right) d s\right)\left[S_{h}(0)\right. \\
& \quad+\int_{0}^{t}\left(\Lambda+I_{h}(s)+\gamma R_{h}(s)\right) \\
& \left.\cdot \exp \left(\int_{0}^{s}\left(k_{h}(c)+d_{h}\right) d c\right) d s\right] \\
& \quad \geq \exp \left(-\int_{0}^{t}\left(k_{h}(s)+d_{h}\right) d s\right) \\
& \cdot\left[\int_{0}^{t}\left(\Lambda+I_{h}(s)+\gamma R_{h}(s)\right)\right. \\
& \left.\cdot \exp \left(\int_{0}^{s}\left(k_{h}(c)+d_{h}\right) d c\right) d s\right]>0, \quad \forall t>0,
\end{aligned}
$$




$$
\begin{gathered}
E_{h}(t)=e^{-\left(d_{h}+\alpha\right) t}\left(E_{h}(0)+\int_{0}^{t} k_{h}(s) S_{h}(s) e^{\left(d_{h}+\alpha\right) s} d s\right) \\
\geq e^{-\left(d_{h}+\alpha\right) t}\left(\int_{0}^{t} k_{h}(s) S_{h}(s) e^{\left(d_{h}+\alpha\right) s} d s\right)>0, \\
I_{h}(t)=e^{-\left(d_{h}+d_{p}+r_{h}\right) t}\left(I_{h}(0)+\int_{0}^{t} \alpha E_{h}(s) e^{\left(d_{h}+d_{p}+r_{h}\right) s} d s\right) \\
\geq e^{-\left(d_{h}+d_{p}+r_{h}\right) t}\left(\int_{0}^{t} \alpha E_{h}(s) e^{\left(d_{h}+d_{p}+r_{h}\right) s} d s\right)>0, \\
R_{h}(t)=e^{-\left(d_{h}+\gamma\right) t}\left(R_{h}(0)+\int_{0}^{t} r_{h} I_{h}(s) e^{\left(d_{h}+\gamma\right) s} d s\right) \\
\geq e^{-\left(d_{h}+\gamma\right) t}\left(\int_{0}^{t} r_{h} I_{h}(s) e^{\left(d_{h}+\gamma\right) s} d s\right)>0, \quad \forall t>0, \\
\left.I_{m}(t)=e^{-d_{m}^{s}\left(k_{m}(c)+d_{m}+v_{m}\right) d c} d s\right]>0, \forall t>0, \quad \forall t>0 . \\
E_{m}(t)=e^{\int_{0}^{t}-\left(k_{m}(s)+d_{m}+v_{m}\right) d s}\left[\int_{m}^{t} E_{m}(0)+\int_{0}^{t} k_{m}(s)\right. \\
\left.\left(A(s)-I_{m}(s)\right) e^{\int_{0}^{s}\left(k_{m}(c)+d_{m}+v_{m}\right) d c} d s\right]
\end{gathered}
$$

Thus, $X_{0}$ is positively invariant. So, $\partial X_{0}$ is also positively invariant.

Note that, from Theorem $2, \Omega$ is a compact set which attracts all positive orbits in $X$, which implies that the discrete-time system $P: X \rightarrow X$ is point dissipative. Moreover, $\forall n_{0} \geq 1, P^{n_{0}}$ is compact; it then follows from Theorem 2.9 in [15] that $P$ admits a global attractor in $X$.

Lemma 13. If $\mathscr{R}_{0}>1$, there exists $\eta>0$ such that when $\| \psi-$ $E_{1}^{+} \| \leq \eta, \forall \psi \in X_{0}$, we have lim $\sup _{m \rightarrow \infty}\left\|P^{m}(\psi)-E_{1}^{+}\right\| \geq \eta$.

Proof. Suppose by contradiction that $\limsup _{m \rightarrow \infty} \| P^{m}(\psi)-$ $E_{1}^{+} \|<\eta$ for some $\psi \in X_{0}$. Then, there exists an integer $n \geq 1$ such that, for all $m \geq n,\left\|P^{m}(\psi)-M\right\|<\eta$. By the continuity of the solution $u(t, \psi)$, we have $\left\|u\left(t, P^{m}(\psi)\right)-u\left(t, E_{1}^{+}\right)\right\| \leq \sigma$ for all $t \geq 0$ and $\sigma>0$. For all $t \geq 0$, let $t=m \omega+t_{1}$, where $t_{1} \in[0, \omega]$ and $m=[t / \omega]$. [t/ $\left.\omega\right]$ is the greatest integer less than or equal to $t / \omega$. If $\left\|\psi-E_{1}^{+}\right\| \leq \eta$, then by the continuity of the solution $u(t, \psi)$ we have

$$
\begin{aligned}
& \left\|u(t, \psi)-u\left(t, E_{1}^{+}\right)\right\| \\
& \quad=\left\|u\left(t_{1}+m \omega, \psi\right)-u\left(t_{1}+m \omega, M\right)\right\| \\
& \quad=\left\|\Phi\left(t_{1}+m \omega\right) \psi-\Phi\left(t_{1}+m \omega\right) E_{1}^{+}\right\| \\
& =\left\|\Phi\left(t_{1}\right) \Phi(m \omega) \psi-\Phi\left(t_{1}\right) \Phi(m \omega) E_{1}^{+}\right\| \\
& =\left\|\Phi\left(t_{1}\right) P^{m}(\psi)-\Phi\left(t_{1}\right) P^{m}\left(E_{1}^{+}\right)\right\| \\
& =\left\|\Phi\left(t_{1}\right) P^{m}(\psi)-\Phi\left(t_{1}\right) E_{1}^{+}\right\| \leq \sigma .
\end{aligned}
$$

It then follows that $S_{h}^{*}-\sigma \leq S_{h}(t) \leq S_{h}^{*}+\sigma$ and $A^{*}-\sigma \leq A(t) \leq$ $A^{*}+\sigma$. So, there exists $\sigma^{*}>0$ such that $S_{h}(t) / N_{h}(t) \geq 1-\sigma^{*}$ and $A(t) / N_{h}(t) \geq A^{*} / N_{h}^{*}-\sigma^{*}$.

From (11) we have

$$
\begin{aligned}
& \frac{d E_{h}}{d t}(t) \geq c_{m h} \beta(t)\left(1-\sigma^{*}\right) I_{m}(t)-\left(d_{h}+\alpha\right) E_{h}(t), \\
& \frac{d I_{h}}{d t}(t)=\alpha E_{h}(t)-\left(d_{p}+d_{h}+r_{h}\right) I_{h}(t), \\
& \frac{d R_{h}}{d t}(t)=r_{h} I_{h}(t)-\left(d_{h}+\gamma\right) R_{h}(t), \\
& \frac{d E_{m}}{d t}(t) \geq \beta(t)\left(\frac{A^{*}}{N_{h}^{*}}-\sigma^{*}\right)\left[c_{h m} I_{h}(t)+\bar{c}_{h m} R_{h}(t)\right] \\
& \quad-\left(v_{m}+d_{m}\right) E_{m}(t), \\
& \frac{d I_{m}}{d t}(t)=v_{m} E_{m}(t)-d_{m} I_{m}(t) .
\end{aligned}
$$

Let us consider the following auxiliary linear system:

$$
\begin{aligned}
\frac{d \widehat{h}}{d t}(t)= & M_{\sigma^{*}}(t) \widehat{h}(t) ; \\
& \widehat{h}(t)=\left(\widehat{E}_{h}(t), \widehat{I}_{h}(t), \widehat{R}_{h}(t), \widehat{E}_{m}(t), \widehat{I}_{m}(t)\right)^{T}
\end{aligned}
$$

with

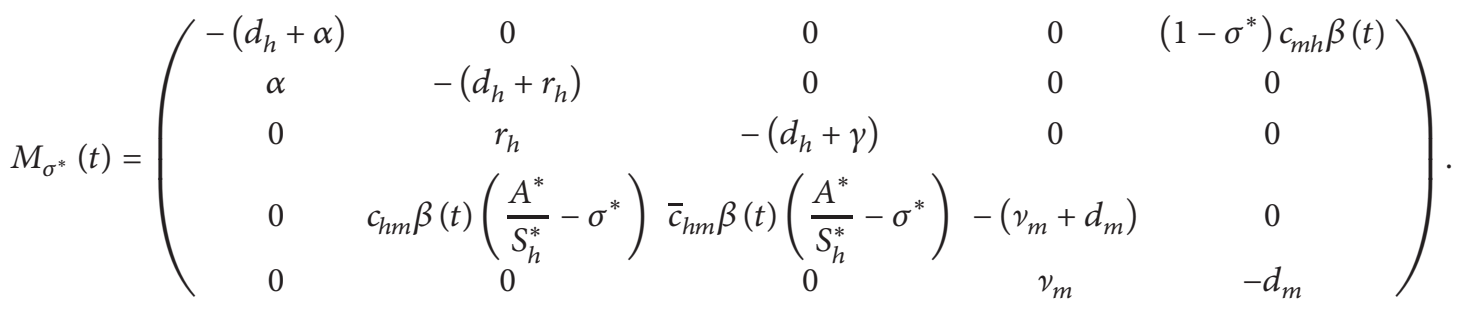


By applying the same method as above, if $\mathscr{R}_{0}>1$ then $\rho\left(\Phi_{M_{\sigma^{*}}}(\omega)\right)>1$. In this case $\theta$ is positive, and then $\widehat{h}(t) \rightarrow \infty$ as $t \rightarrow \infty$. Moreover, since $X_{0}$ is positively invariant, then there exists an integer $q \geq n$ and a real number $\kappa>0$ such that

$$
\begin{aligned}
& \left(E_{h}(q \omega), I_{h}(q \omega), R_{h}(q \omega), E_{m}(q \omega), I_{m}(q \omega)\right) \\
& \quad \geq \kappa \widehat{h}(0) .
\end{aligned}
$$

Applying the theorem of comparison principle, we get

$$
\begin{gathered}
\left(E_{h}(q \omega+t), I_{h}(q \omega+t), R_{h}(q \omega+t), E_{m}(q \omega+t),\right. \\
\left.I_{m}(q \omega+t)\right) \geq \kappa \widehat{h}(t), \quad \forall t \geq 0 .
\end{gathered}
$$

It then follows that $\lim _{t \rightarrow \infty}\left|E_{h}(t), I_{h}(t), R_{h}(t), E_{m}(t), I_{m}(t)\right|=$ $\infty$, which contradicts the fact that solutions are bounded.

Theorem 14. If $\mathscr{R}_{0}>1$, then system (7) has at least one positive periodic solution.

Proof. We first prove that $P$ is uniformly persistent with respect to $\left(X_{0}, \partial X_{0}\right)$.

We define the following sets:

$$
\begin{aligned}
M_{\partial} & =\left\{\psi \in \partial X_{0} \mid P^{m}(\psi) \in \partial X_{0}, \text { for any } m \geq 0\right\}, \\
\mathscr{D} & =\left\{\left(S_{h}, 0,0,0,0,0\right) \in X \mid S_{h} \geq 0\right\} .
\end{aligned}
$$

Let us prove that $M_{\partial}=\mathscr{D}$.

It is easy to remark that $\mathscr{D} \subset M_{\partial}$. We only need to prove that $M_{\partial} \subset \mathscr{D}$.

Let $\psi \in \partial X_{0} \backslash \mathscr{D}$. If

(i) $I_{h}(0)>0, I_{m}(0)>0$, and $E_{h}(0)=E_{m}(0)=R_{h}(0)=0$, then we have $S_{h}(t)>0, I_{h}(t)>0, I_{m}(t)>0, E_{m}(t)>0$, $E_{h}(t)>0, R_{h}(t)>0, \forall t>0$,

(ii) $I_{h}(0)=I_{m}(0)=0$ and $E_{h}(0)>0, E_{m}(0)>0, R_{h}(0)>$ 0 , then we have $S_{h}(t)>0, I_{h}(t)>0, I_{m}(t)>0, E_{m}(t)>$ $0, E_{h}(t)>0, R_{h}(t)>0, \forall t>0$.

For any cases, it follows that $\left(S_{h}(t), E_{h}(t), I_{h}(t), R_{h}(t), E_{m}(t)\right.$, $\left.I_{m}(t)\right) \notin \partial X_{0}$ for $t>0$ sufficiently small, which contradicts the fact that $\partial X_{0}$ is positively invariant. Hence, $M_{\partial} \subset \mathscr{D}$. Thus, it then follows that $M_{\partial}=\mathscr{D}$.

The equality $M_{\partial}=\mathscr{D}$ implies that $E_{1}^{+}$is a fixed point of $P$ and acyclic in $M_{\partial}$; every solution in $M_{\partial}$ approaches to $E_{1}^{+}$. Moreover, Lemma 13 implies that $E_{1}^{+}$is an isolated invariant set in $X$ and $W^{s}\left(E_{1}^{+}\right) \cap X_{0}=\emptyset$. By the acyclicity theorem on uniform persistence for maps, Theorem 1.3.1 and Remark 1.3.1 in [14], it follows that $P$ is uniformly persistent with respect to $X_{0}$. Thus, Theorem 3.1.1 in [14] implies that the periodic semiflow $\Phi(t): X \rightarrow X$ is also uniformly persistent with respect to $X_{0}$. Thanks to Theorem 1.3.6 in [14], model (11) has at least one $\omega$-periodic solution $\widetilde{u}\left(t, \psi^{*}\right)$ with $\psi^{*} \in X_{0}$ and $t \geq 0$. Now, we show that $\widetilde{u}\left(t, \psi^{*}\right)$ is positive.

Suppose that $\psi^{*}=0$; then, for all $t>0$, we obtain $\widetilde{u}_{i}\left(t, \psi^{*}\right)>0$, for $i=1,2,3,4,5,6$. By using the periodicity of the solution, we have $S_{h}^{*}(0)=S_{h}^{*}(n \omega)=0, E_{h}^{*}(0)=E_{h}^{*}(n \omega)=$ $0, I_{h}^{*}(0)=I_{h}^{*}(n \omega)=0, R_{h}^{*}(0)=R_{h}^{*}(n \omega)=0, E_{m}^{*}(0)=$ $E_{m}^{*}(n \omega)=0, I_{m}^{*}(0)=I_{m}^{*}(n \omega)=0, \forall n \geq 1$, which contradicts the fact that $\widetilde{u}_{i}\left(t, \psi^{*}\right)>0$ for $i=1,2,3,4,5,6$. So, the periodic solution is positive.

\section{Numerical Simulation}

In this section, we will present a series of numerical simulations of model (11) in order to support our theoretical results, to predict the trend of the disease, and to explore some control measures.

4.1. Initial Conditions and Estimation of $\beta(t)$. To validate our results, we choose the following initial conditions: $E(0)=$ 2400, $L(0)=1200, S_{h}(0)=1500, E_{h}(0)=50, I_{h}(0)=200$, $R_{h}(0)=50, S_{m}(0)=3000, E_{m}(0)=100, I_{m}(0)=500$, and $A(0)=3600$. Our numerical simulation will be performed using the MATLAB technical computing software with the fourth-order Runge-Kutta method [16].

Using the method developed in [11], we express the biting rate as follows:

$$
\begin{aligned}
\beta(t)= & \alpha_{0}-1.83692 \cos (0.523599 t) \\
& -0.175817 \cos (1.0472 t) \\
& -0.166233 \cos (1.5708 t) \\
& -0.16485 \cos (2.0944 t) \\
& -0.17681 \cos (2.61799 t) \\
& -1.37079 \sin (0.523599 t) \\
& +0.296267 \sin (1.0472 t) \\
& +0.2134 \sin (1.5708 t) \\
& -0.295228 \sin (2.0944 t) \\
& -0.201712 \sin (2.61799 t)
\end{aligned}
$$

with $\alpha_{0} \geq 3$.

4.2. The Model Parameters and Their Dimensions. Numerical values of parameters are given in Table 1 .

4.3. Numerical Results. Using the above initial conditions, we now simulate model (11) in order to illustrate our mathematical results.

By taking $\alpha_{0}=7, d_{p}=0.0028, c_{m h}=0.022, c_{h m}=0.48$, $\bar{c}_{h m}=0.048, b=180, s=15, d=6, d_{L}=7.5, s_{L}=15$, $d_{m}=3.4038$ and considering the above initial conditions, we get $r=25.1819, \mathscr{R}_{0}=1.3310>1$ and Figures 2, 3, and 4 .

Figure 2 describes the evolution of infected (exposed and infectious) humans. Figure 3 describes the evolution of infected (exposed and infectious) mosquitoes and Figure 4 describe the evolution of susceptible humans and mosquitoes. Figures 2 and 3 show that malaria remains 
TABLE 1: Values for constant parameters for the malaria model.

\begin{tabular}{|c|c|c|c|c|}
\hline Parameter & Description & Value & Reference & Dimension \\
\hline$\Lambda$ & Constant recruitment rate for humans & 400 & Estimated & Humans/month \\
\hline$d_{h}$ & Human death rate & 0.019 & Estimated & /month \\
\hline$\alpha$ & Transmission rate of humans from $E_{h}$ to $I_{h}$ & 3.04 & {$[17]$} & /month \\
\hline$d_{p}$ & Disease-induced death rate for humans & 0.0028 & {$[11]$} & /month \\
\hline$r_{h}$ & Recovery rate of humans & 0.0159 & {$[11]$} & /month \\
\hline$\gamma$ & Per capita rate of loss of immunity for humans & 0.0167 & {$[11]$} & /month \\
\hline$s_{L}$ & Transfer rate from $L$ to adult & 15 & {$[6]$} & /month \\
\hline$d_{m}$ & Death rate for adult vectors & 3.4038 & {$[11]$} & /month \\
\hline$v_{m}$ & Transmission rate of mosquitoes from $E_{m}$ to $I_{m}$ & 2.523 & {$[11]$} & /month \\
\hline$c_{m h}$ & Probability of transmission of infection from $I_{m}$ to $S_{h}$ & 0.022 & {$[17]$} & Dimensionless \\
\hline$c_{h m}$ & Probability of transmission of infection from $I_{h}$ to $S_{m}$ & 0.48 & {$[17]$} & Dimensionless \\
\hline $\bar{c}_{h m}$ & Probability of transmission of infection from $R_{h}$ to $S_{m}$ & 0.048 & {$[17]$} & Dimensionless \\
\hline$K_{E}$ & Available breeder sites occupied by eggs & 30000 & Estimated & Space \\
\hline$K_{L}$ & Available breeder sites occupied by larvae & 18000 & Estimated & Space \\
\hline$s$ & Transfer rate from $E$ to $L$ & 15 & {$[6]$} & /month \\
\hline$b$ & Eggs laying rate & 180 & {$[6]$} & /month \\
\hline$d$ & Death rate of eggs & 6 & {$[6]$} & /month \\
\hline$d_{L}$ & Larvae death rate & 6 & {$[6]$} & /month \\
\hline
\end{tabular}

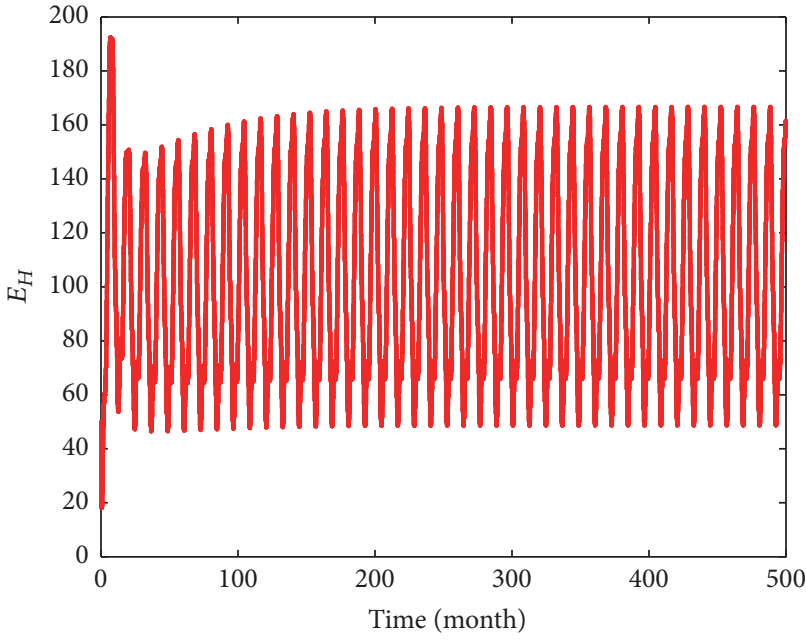

- Exposed humans

(a)

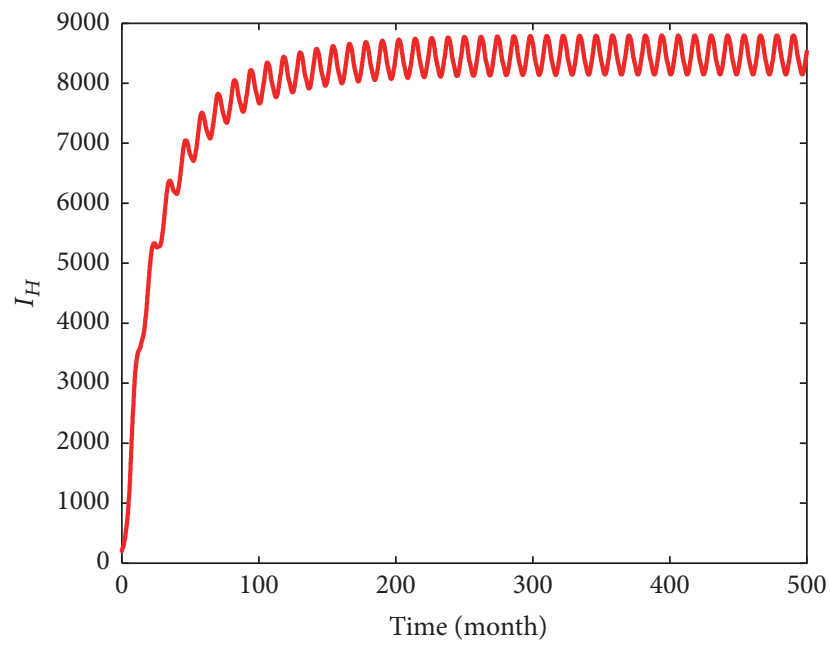

- Infectious humans

FIGURE 2: Distribution of infected humans.

persistent in the two populations. Besides, we observe that system (11) has one positive periodic solution. So, these numerical results illustrate the result of our Theorem 14 .

In order to understand the model behaviour around the disease-free equilibrium, we consider the same above initial conditions and the following values: $\alpha_{0}=4, d_{p}=0, c_{m h}=$ $0.022, c_{h m}=0.24, \bar{c}_{h m}=0.024, b=180, s=15, d=6$,
$d_{L}=7.5, s_{L}=15, d_{m}=6$. Then we get $r=14.2857$ and $\mathscr{R}_{0}=$ $0.2602<1$. Figures 5 and 6 illustrate that the disease dies out in both populations. Thus, the numerical results are the same as what we got in Theorem 11 .

4.4. Parameters of Control of Malaria. Now, we assume that people became more conscious about the malaria disease and 


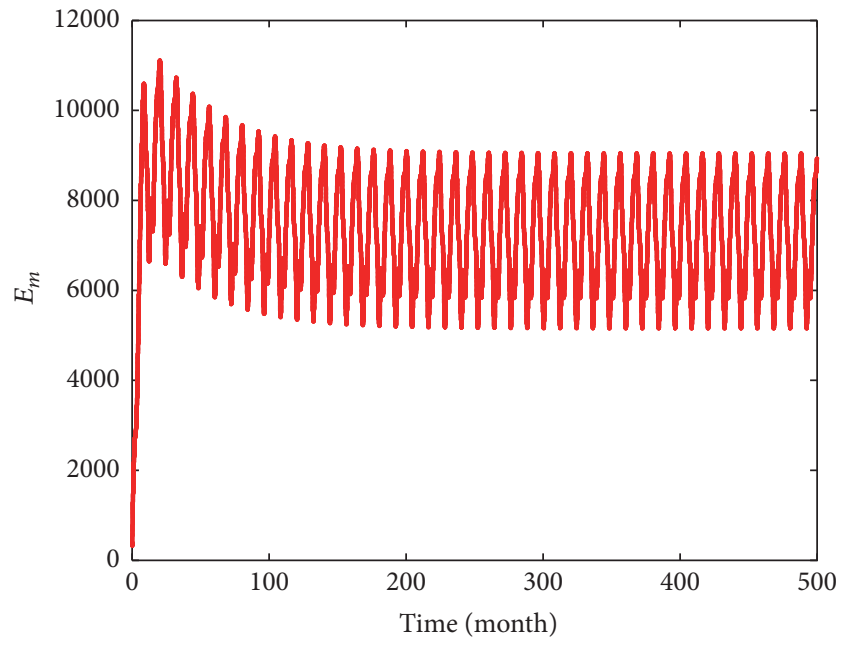

- Exposed mosquitoes

(a)

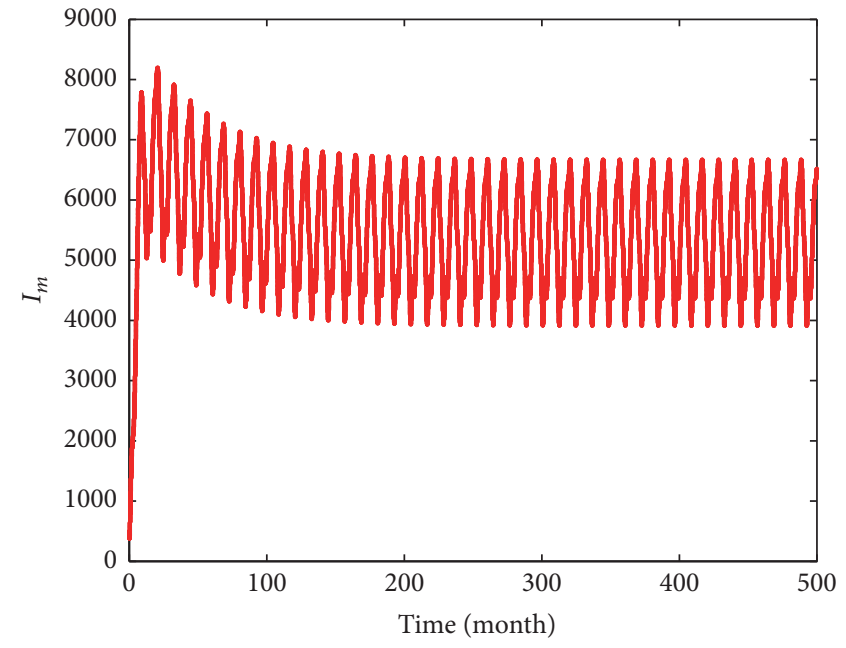

- Infectious mosquitoes

(b)

FIGURE 3: Distribution of infected mosquitoes.

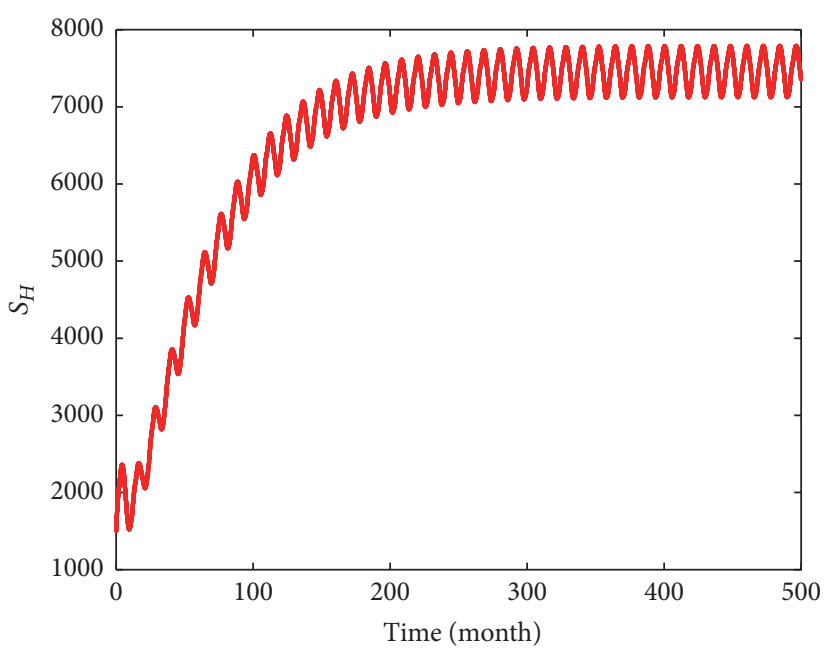

- Susceptible humans

(a)

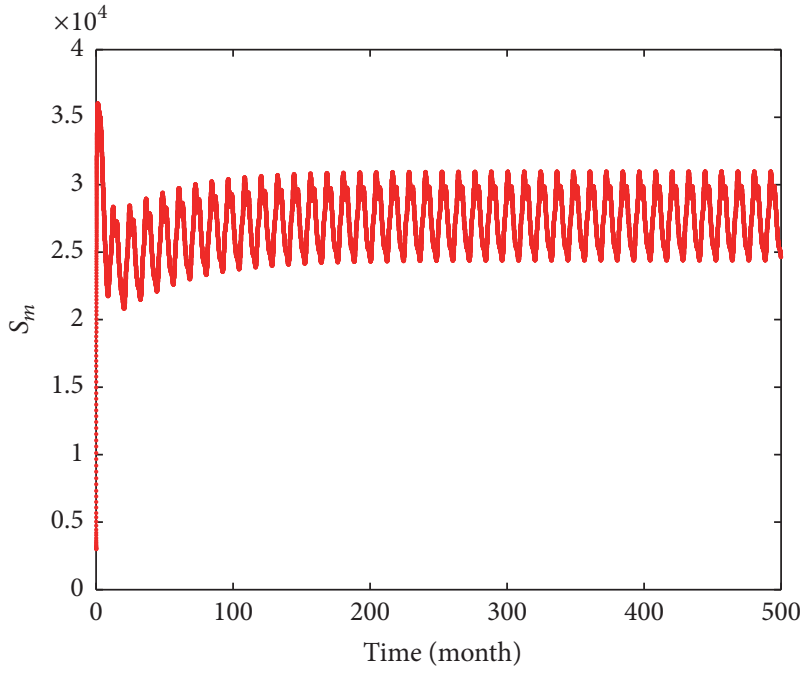

- Susceptible mosquitoes

(b)

FIgURE 4: Distribution of susceptible humans and mosquitoes.

they use some efficient methods to reduce the proliferation of mosquitoes. That reduction can perhaps consist in fighting against the development of eggs, larvae, and pupa, firstly, by using chemical application methods (larvicide) or by introducing larvivore fish, and secondly, by using ecological methods (cleaning up the environment) to reduce the breeding sites of eggs and larvae. Let $\mu_{1}, \mu_{2} \in[0,1[$, respectively, be the efficiency of both intervention measures. So, we will use $\widetilde{r}=\left(1-\mu_{1}\right) r, \widetilde{K}_{E}=\left(1-\mu_{2}\right) K_{E}$, and $\widetilde{K}_{L}=\left(1-\mu_{2}\right) K_{L}$ in order to evaluate their impact on the dynamics of malaria transmission.

Thus, by considering the above initial conditions and by taking $\alpha_{0}=7, d_{p}=0.0028, c_{m h}=0.022, c_{h m}=0.48, \bar{c}_{h m}=$ $0.048, d_{m}=3.4038$, we obtain the following results.

(i) Numerical Results for $\mu_{1} \simeq 89 \%$. For this value, we get $\tilde{r}=2.8204$ and $\mathscr{R}_{0}=0.6414$. Moreover, according to Figure 7, we notice that the distribution of infected humans 


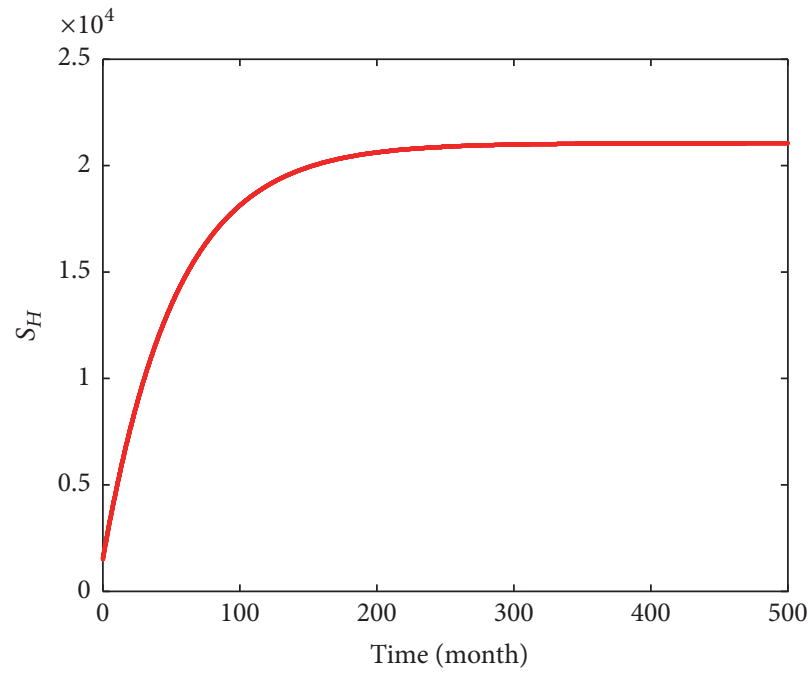

- Susceptible humans

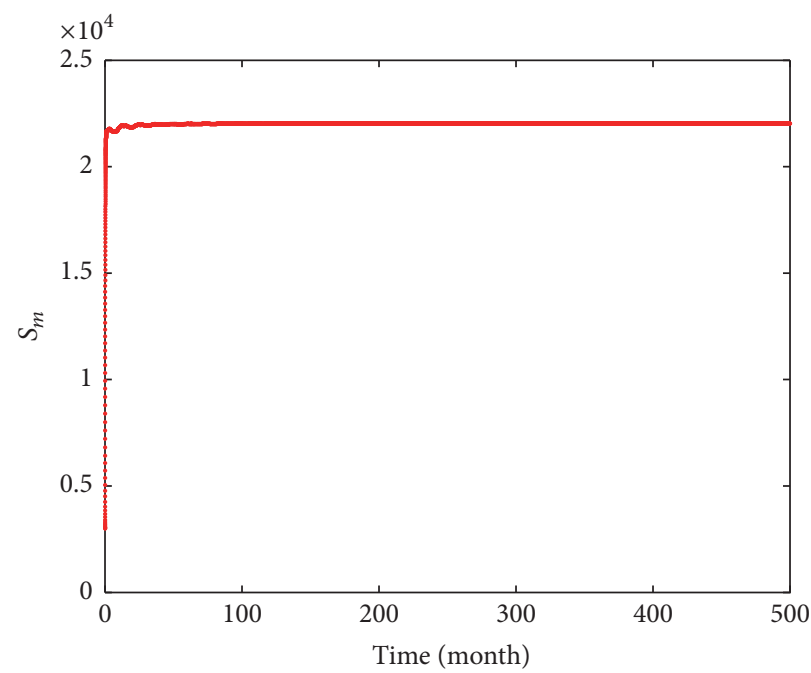

- Susceptible mosquitoes

FIGURE 5: Distribution of susceptible humans and mosquitoes.

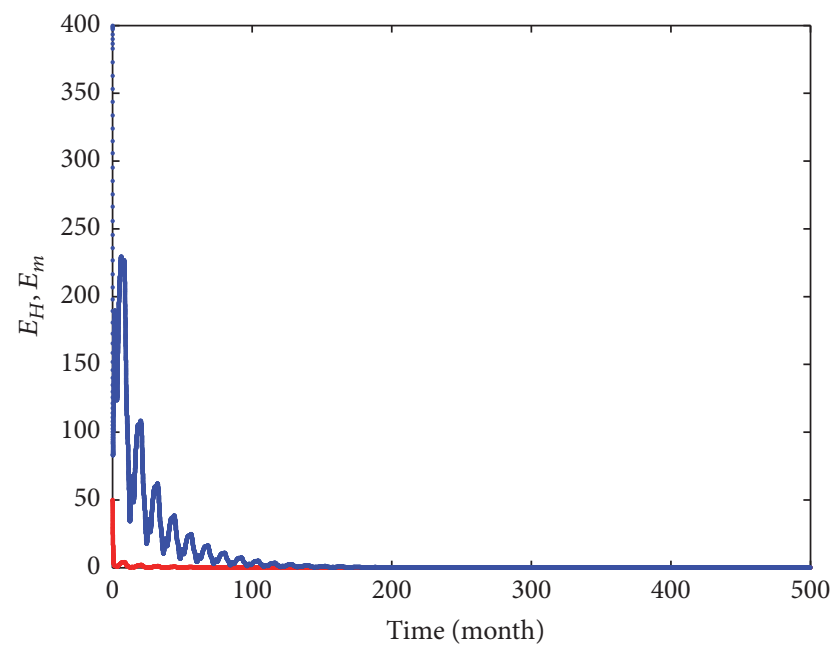

- Exposed humans

- Exposed mosquitoes

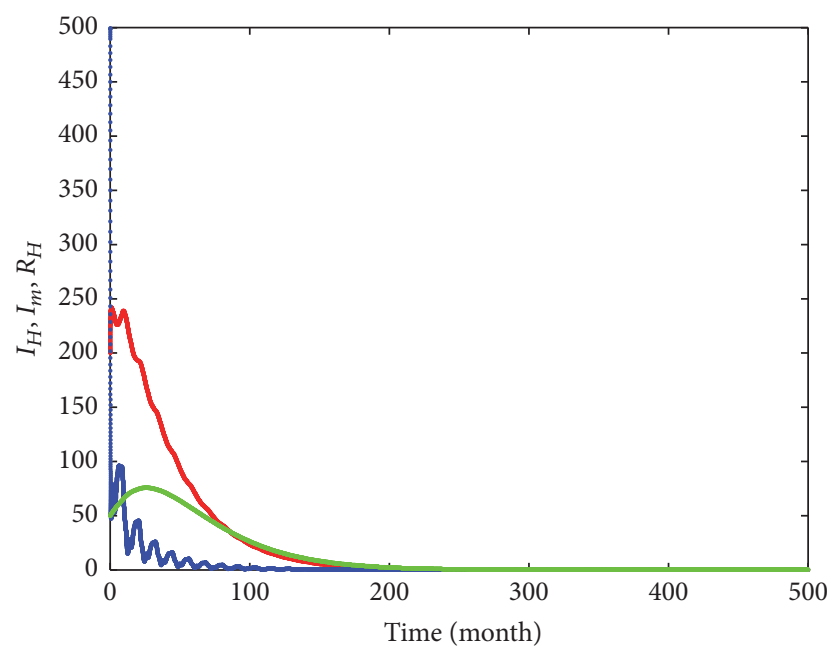

- Infectious humans

- Infectious mosquitoes

- Recovered humans

FIGURE 6: Distribution of infected humans and mosquitoes.

and mosquitoes has highly reduced and the malaria is progressively dying out in the populations.

(ii) Numerical Results for $\mu_{2}=80 \%$. Using $\mu_{2}=0.8$, we get $\widetilde{K}_{E}=6000, \widetilde{K}_{L}=3600$, and $\mathscr{R}_{0}=0.5953$. Further, Figure 8 clearly shows that the disease is quickly disappearing from the populations.

Remark 15. We must notice that the two parameters are important in the malaria transmission because a little perturbation of those parameters influences the dynamics of malaria transmission. So they can be used to fight against the persistence of the disease. The control $\mu_{1}$ is efficient but its action is very slow in finite time, but the control $\mu_{2}$ is the best because it is more optimal and its action is very quick. Thus cleaning up the environment can be a very good mean of controlling malaria in the populations.

\section{Conclusion}

In this paper, we have presented a seasonal determinist model of malaria transmission. From the theoretical point of view, 

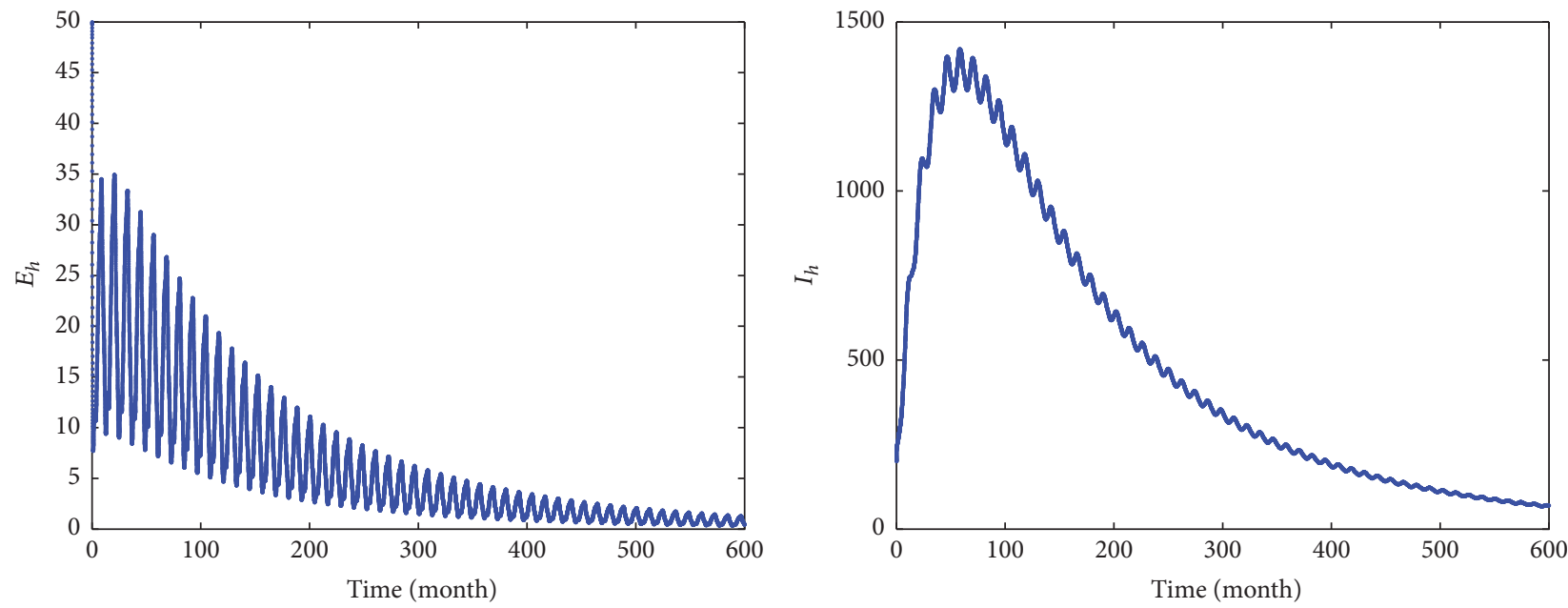

- Exposed humans

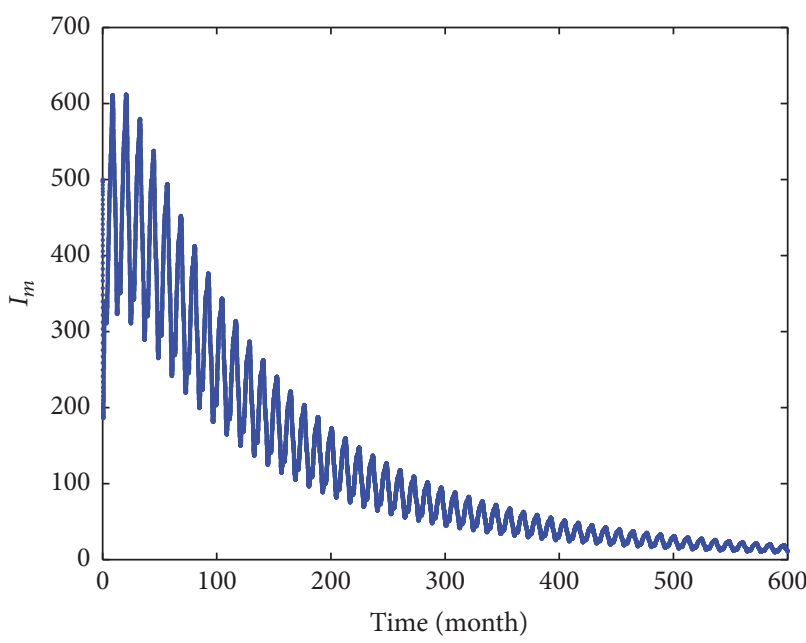

- Infectious humans

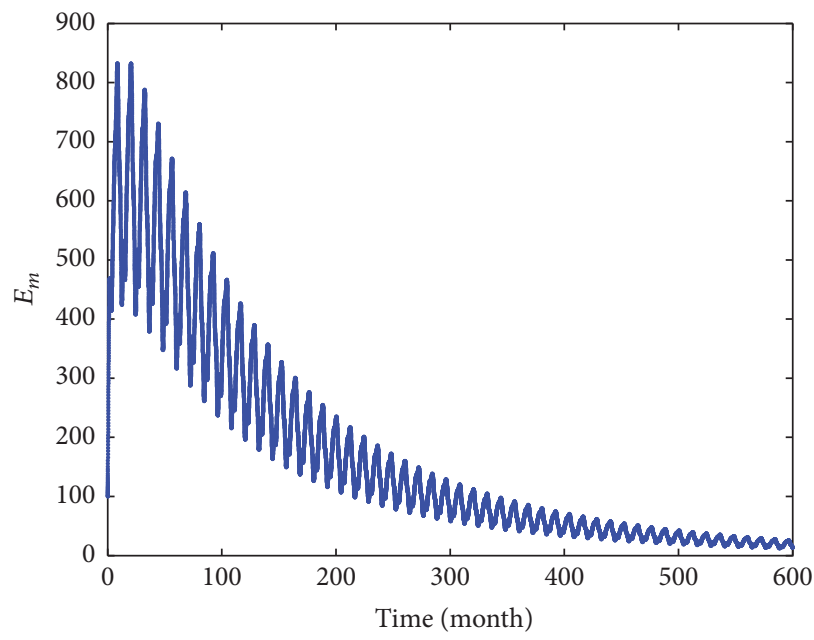

- Infectious mosquitoes

- Exposed mosquitoes

FIgURE 7: Distribution of infected humans and mosquitoes for $b=80, s=10, d=15, s_{L}=6$, and $d_{L}=14$.

we have shown that the basic reproduction ratio, $\mathscr{R}_{0}$, is the distinguishing threshold parameter of the extinction or the persistence of the disease: if $\mathscr{R}_{0}$ is less than 1 malaria disappears in the human and mosquito populations and if it is greater than 1 malaria persists.

It also emerges from our study that the transmission of malaria is highly influenced by the dynamics of immature mosquitoes and depends on the regulatory threshold parameter of the mosquito population, $r$. Thus, the severity of malaria increases with this parameter. So, the life cycle of the anopheles is a very important aspect that must be taken into account in malaria modeling.

Moreover, we have shown that malaria transmission can be controlled by fighting against the proliferation of the mosquitoes, namely, by reducing the value of $r$ or by reducing the value of available breeder sites, $K_{E}$ and $K_{L}$. We have proved that the reduction of the available breeder sites is a very efficient and more ecological method in fighting against malaria transmission. It then follows that environmental sanitation can be a very good means to control malaria in the endemic regions.

However, it must be noticed that our model is limited due to the following reasons: (i) we have not considered the effect of climate change on the life cycle of mosquitoes. (ii) The larva and pupa class were not distinguished.

In the future, one can develop a more realistic model by incorporating the above important factors and by considering the general force of infection. In addition, we can also take into account the degree of vulnerability of human populations in the model. 

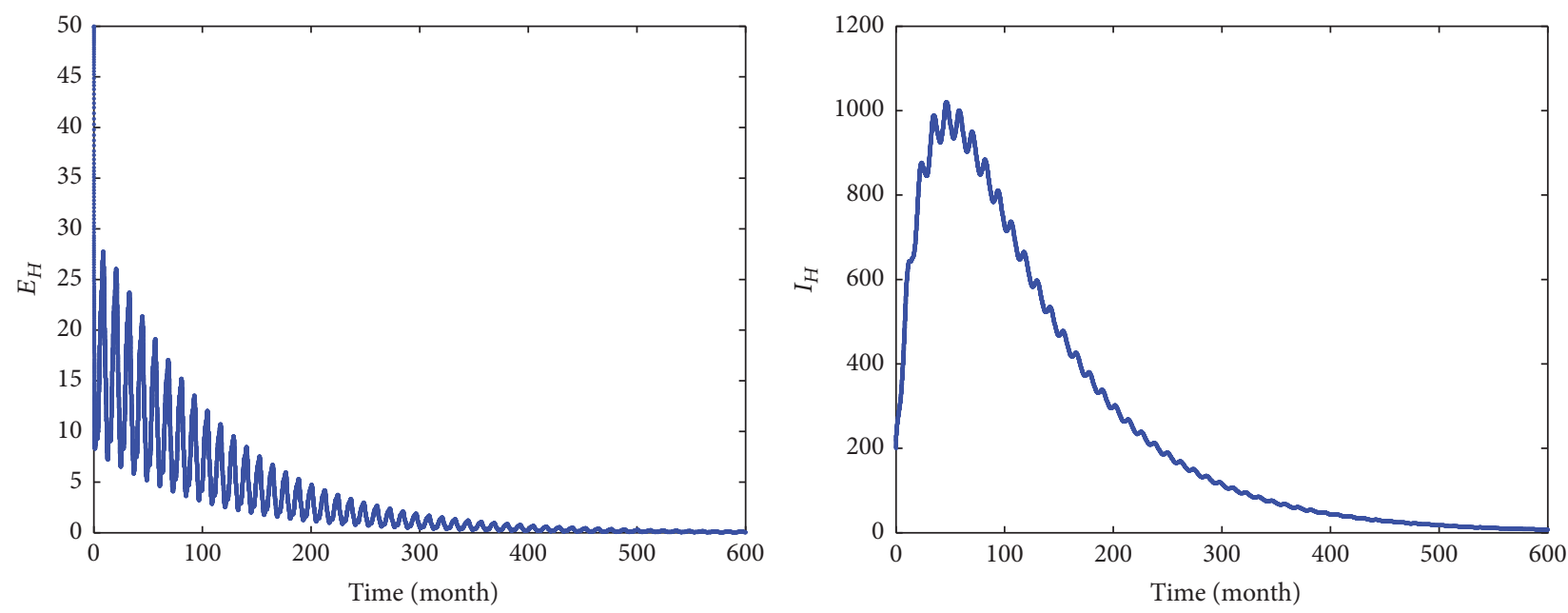

- Exposed humans

- Infectious humans

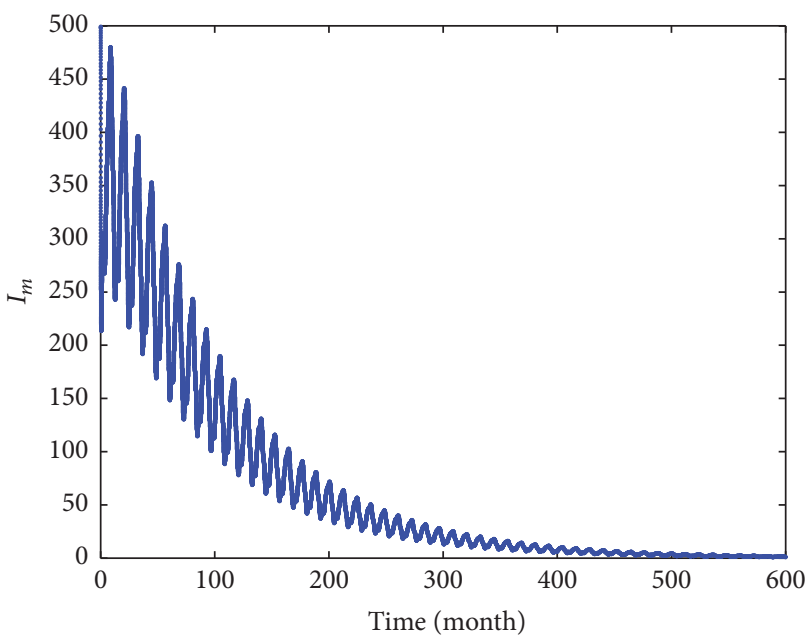

- Infectious mosquitoes

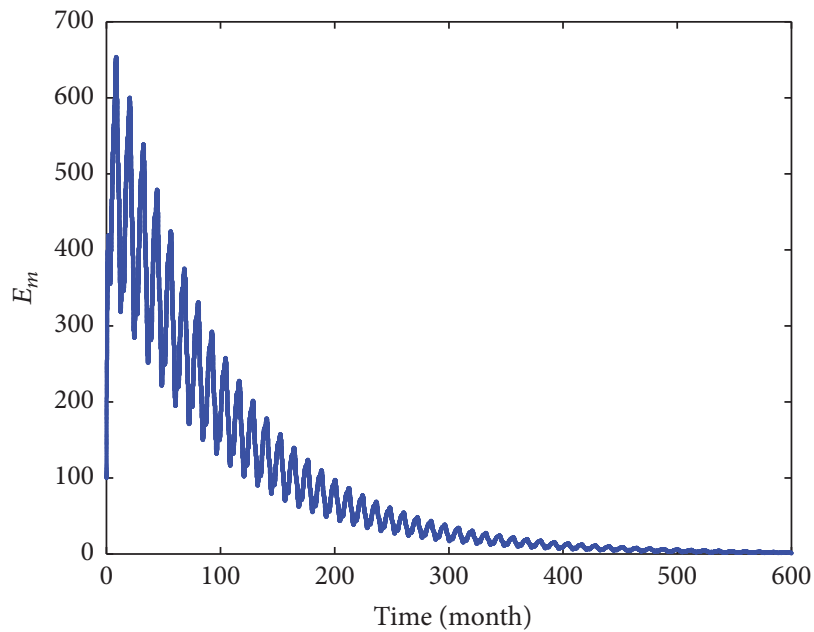

- Exposed mosquitoes

FIGURE 8: Distribution of infected humans and mosquitoes for $b=180, s=15, d=6, s_{L}=15$, and $d_{L}=7.5$.

\section{Conflicts of Interest}

The authors declare that there are no conflicts of interest regarding the publication of this paper.

\section{References}

[1] R. Ross, The prevention of malaria, London, John Murray, 1911.

[2] G. Macdonald, The epidemiology and control of malaria, Oxford University press, London, 1957.

[3] C. Chiyaka, J. M. Tchuenche, W. Garira, and S. Dube, "A mathematical analysis of the effects of control strategies on the transmission dynamics of malaria," Applied Mathematics and Computation, vol. 195, no. 2, pp. 641-662, 2008.

[4] G. A. Ngwa and W. S. Shu, "A mathematical model for endemic malaria with variable human and mosquito populations," Mathematical and Computer Modelling, vol. 32, no. 7-8, pp. 747-763, 2000 .

[5] L. M. Beck-Johnson, W. A. Nelson, K. P. Paaijmans, A. F. Read, M. B. Thomas, and O. N. Bjørnstad, "The effect of temperature on Anopheles mosquito population dynamics and the potential for malaria transmission," PLoS ONE, vol. 8, no. 11, Article ID e79276, 2013.

[6] D. Moulay, M. A. Aziz-Alaoui, and M. Cadivel, "The chikungunya disease: modeling, vector and transmission global dynamics," Mathematical Biosciences, vol. 229, no. 1, pp. 50-63, 2011.

[7] E. N. Chukwu, "On the boundedness and stability properties of solutions of some differential equations of the fifth order," Annali di Matematica Pura ed Applicata. Serie Quarta, vol. 106, pp. 245-258, 1975.

[8] A. S. Sinha, "Stability result of a sixth order non-linear system," vol. 7, pp. 641-643, 1971.

[9] C. Tunç, "On the stability and boundedness of solutions in a class of nonlinear differential equations of fourth order with constant delay," Vietnam Journal of Mathematics, vol. 38, no. 4, pp. 453-466, 2010.

[10] C. Tunç, "New results on the stability and boundedness of nonlinear differential equations of fifth order with multiple 
deviating arguments," Bulletin of the Malaysian Mathematical Sciences Society, vol. 36, no. 3, pp. 671-682, 2013.

[11] Y. Lou and X.-Q. Zhao, "A climate-based malaria transmission model with structured vector population," SIAM Journal on Applied Mathematics, vol. 70, no. 6, pp. 2023-2044, 2010.

[12] W. Wang and X. Zhao, "Threshold dynamics for compartmental epidemic models in periodic environments," Journal of Dynamics and Differential Equations, vol. 20, no. 3, pp. 699-717, 2008.

[13] J. Wang, S. Gao, Y. Luo, and D. Xie, "Threshold dynamics of a huanglongbing model with logistic growth in periodic environments," Abstract and Applied Analysis, vol. 2014, Article ID 841367, 10 pages, 2014.

[14] Z. Xiao-Qiang, CMS Books in mathematics/Ouvrages de mathématiques de la SMC, Springer Verlag, New York, NY, USA, 16th edition, 2003.

[15] P. Magal and X.-Q. Zhao, "Global attractors and steady states for uniformly persistent dynamical systems," SIAM Journal on Mathematical Analysis, vol. 37, no. 1, pp. 251-275, 2005.

[16] W. Ouedraogo, B. Sangaré, and S. Traoré, "Some mathematical problems arising in biological models: a predator-prey model fish-plankton," Journal of Applied Mathematics and Bioinformatics, vol. 5, no. 4, pp. 1-27, 2015.

[17] N. Chitnis, J. M. Hyman, and J. M. Cushing, "Determining important parameters in the spread of malaria through the sensitivity analysis of a mathematical model," Bulletin of Mathematical Biology, vol. 70, no. 5, pp. 1272-1296, 2008. 


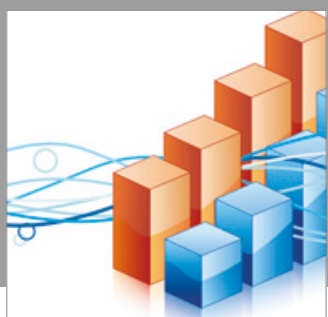

Advances in

Operations Research

vatersals

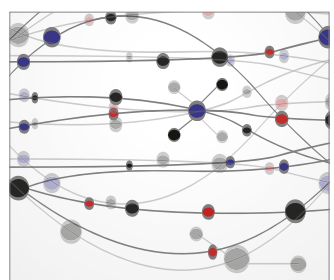

\section{The Scientific} World Journal
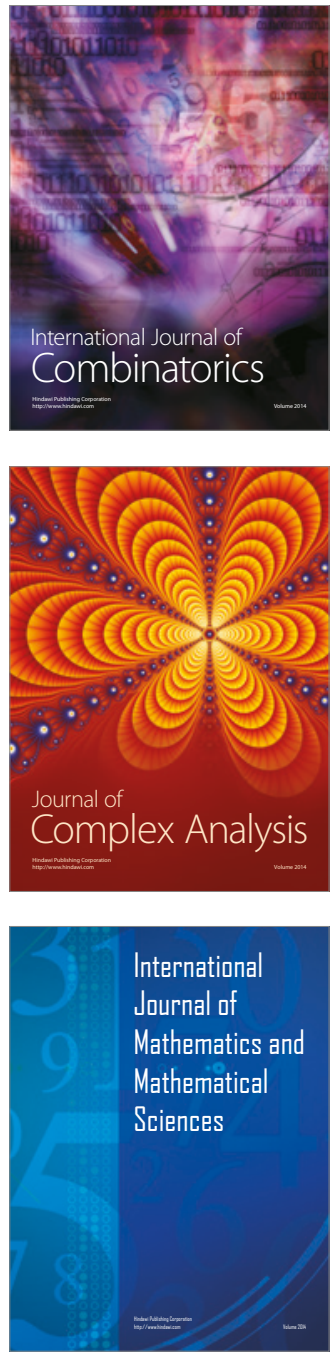
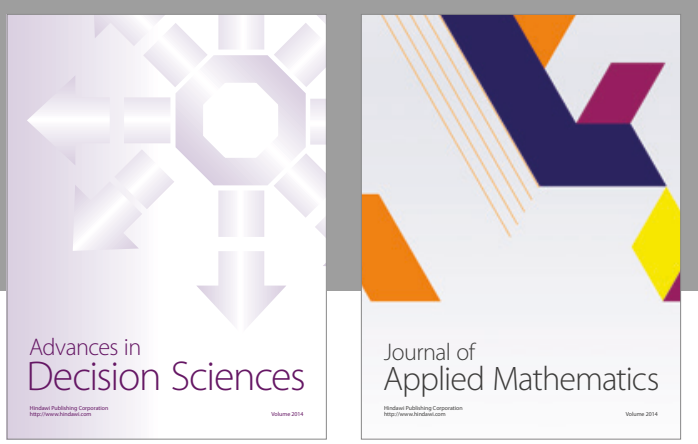

Algebra

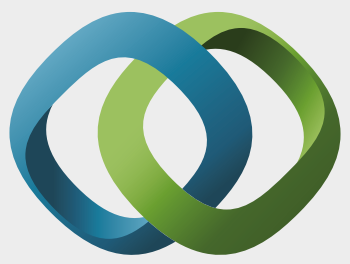

\section{Hindawi}

Submit your manuscripts at

https://www.hindawi.com
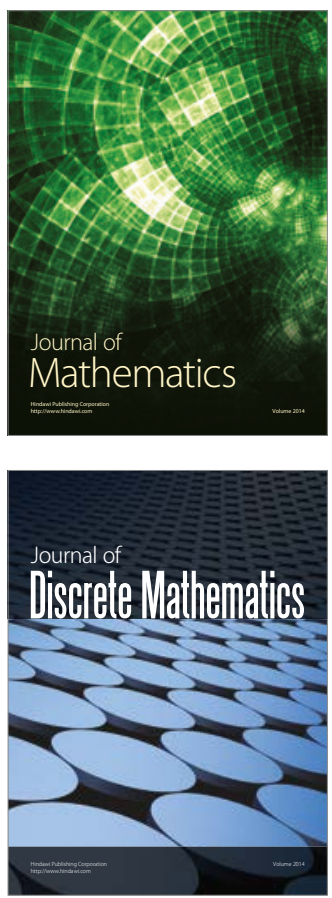

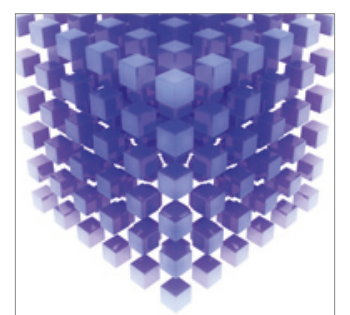

Mathematical Problems in Engineering
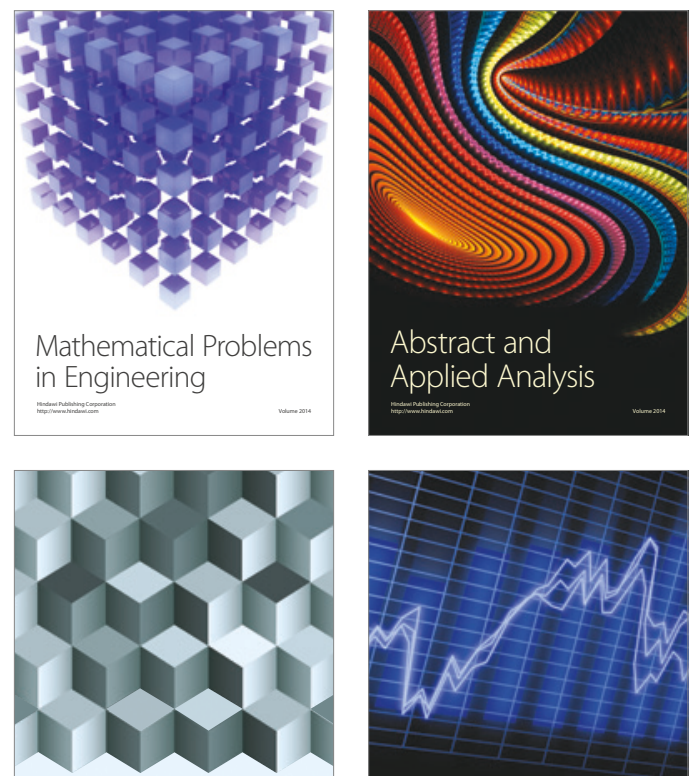

Journal of

Function Spaces

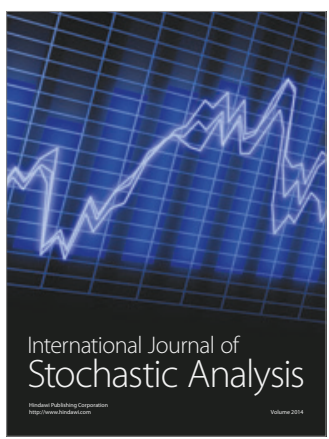

Probability and Statistics
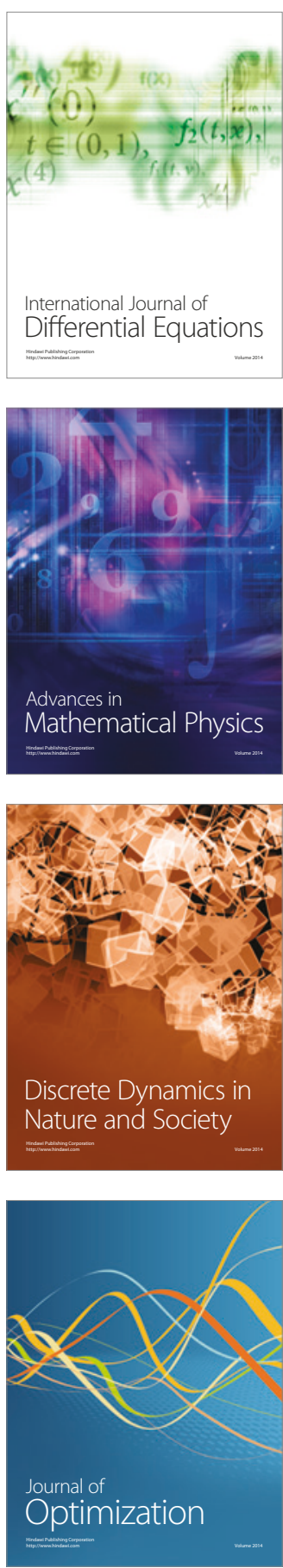